\title{
PICO Results and Future Plans
}

\author{
Hugh Lippincott, Fermilab \\ for the PICO Collaboration \\ EDU 2017
}




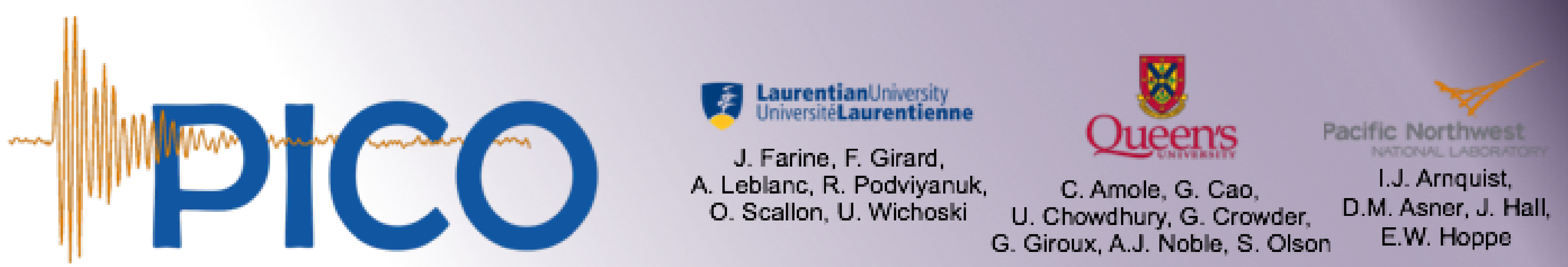

\section{SN-}

K. Clark, I. Lawson

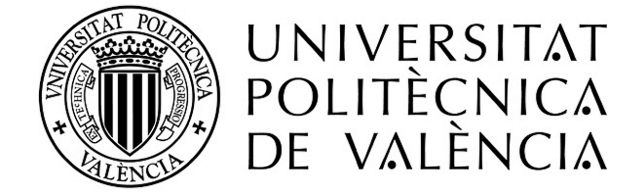

M. Ardid, M. Bou=Cabo, I. Felis

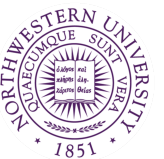

NORTHWESTERN UNIVERSITY

D. Baxter, C.J. Chen, C.E. Dahl, M. Jin, J. Zhang

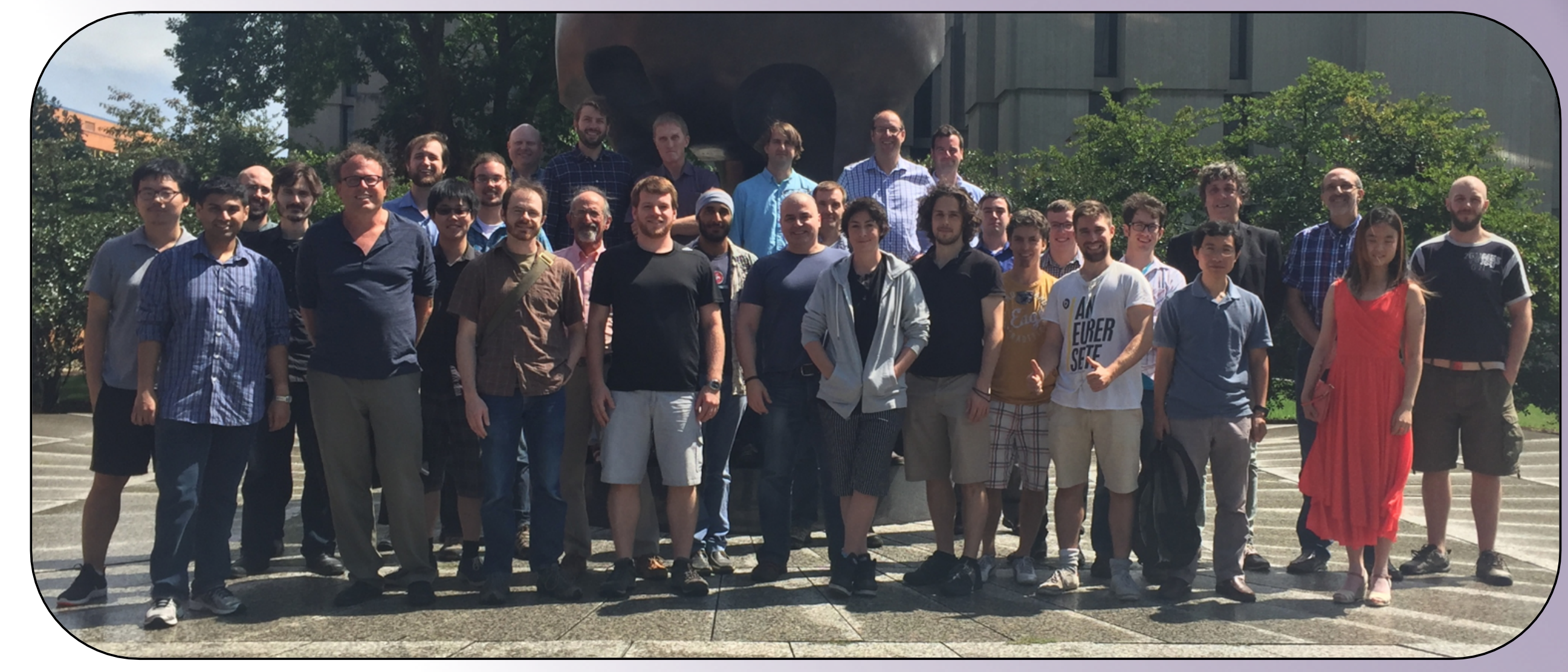

P. Bhattacharjee. M. Das, S. Seth

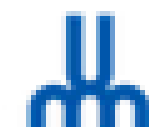

Université $\mathrm{nh}$ de Montréal

M. Laurin, A Plante,

N. Starinski, F. Tardif,

V. Zacek

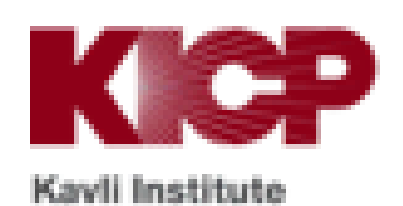

Kavil linsilitute

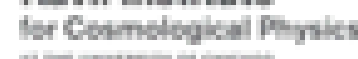
J.l. Collar, A Ortega

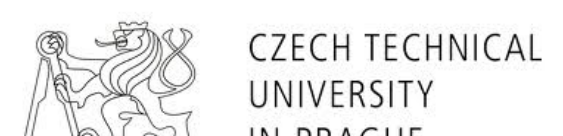

तु IN PRAGUE

R. Filgas, F. Mamedov, I. Stekl
茫Fermilab

P.S. Cooper, M. Crisler, W.H. Lippincott, A.E. Robinson, R. Rucinski, A. Sonnenschein

\section{INDIANA UNIVERSITY SOUTH BEND}

E. Behnke, H. Borsodi, I. Levine, T. Nania, A. Roeder, J. Wells
(2. Drexel

P. Campion, R. Neilson IIIVirginiaTech.

D. Maurya, S. Priya, Y. Yan

$\Gamma$ Northeastern

O. Harris
[9] UHIYERSITY OF MALBERTA

S. Fallows, C. Krauss, P. Mitra
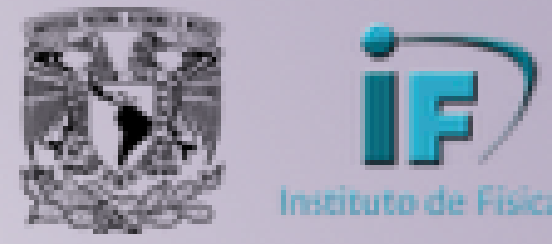

E. Vázquez-Jáuregui 


\section{PICO fast compression bubble chamber}

- Pressure expansion creates superheated fluid, $\mathrm{CF}_{3}$ l or $\mathrm{C}_{3} \mathrm{~F}_{8}$

- I for spin-independent

- F for spin-dependent

- Particle interactions nucleate bubbles

- Cameras see bubbles

- Recompress chamber to reset
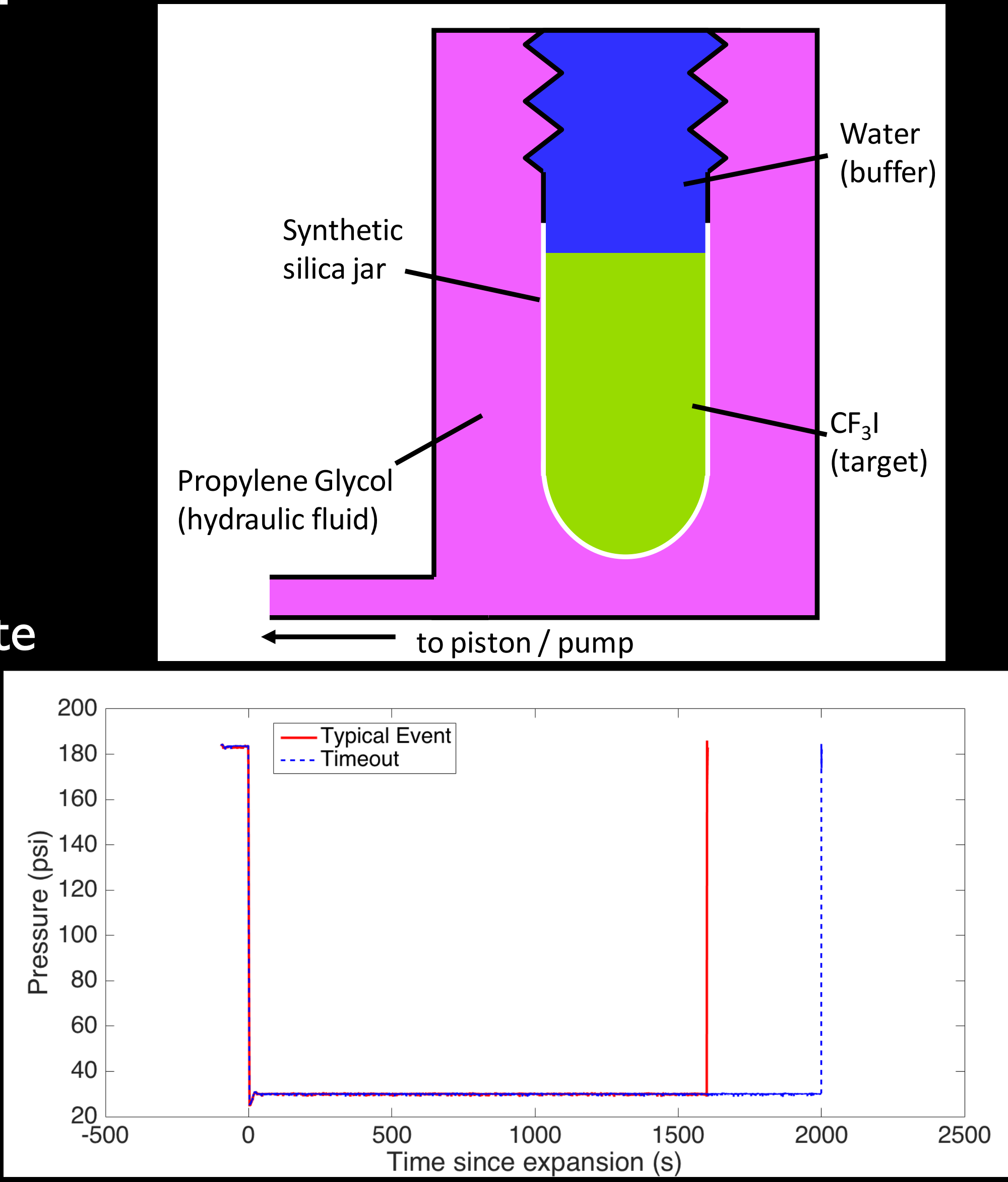


\section{Why bubble chambers?}

- A lot of effort in dark matter experiments goes into discriminating electronic recoils (gammas) vs. nuclear recoils(WIMPs)

Xenon TPCs -

Charge to light
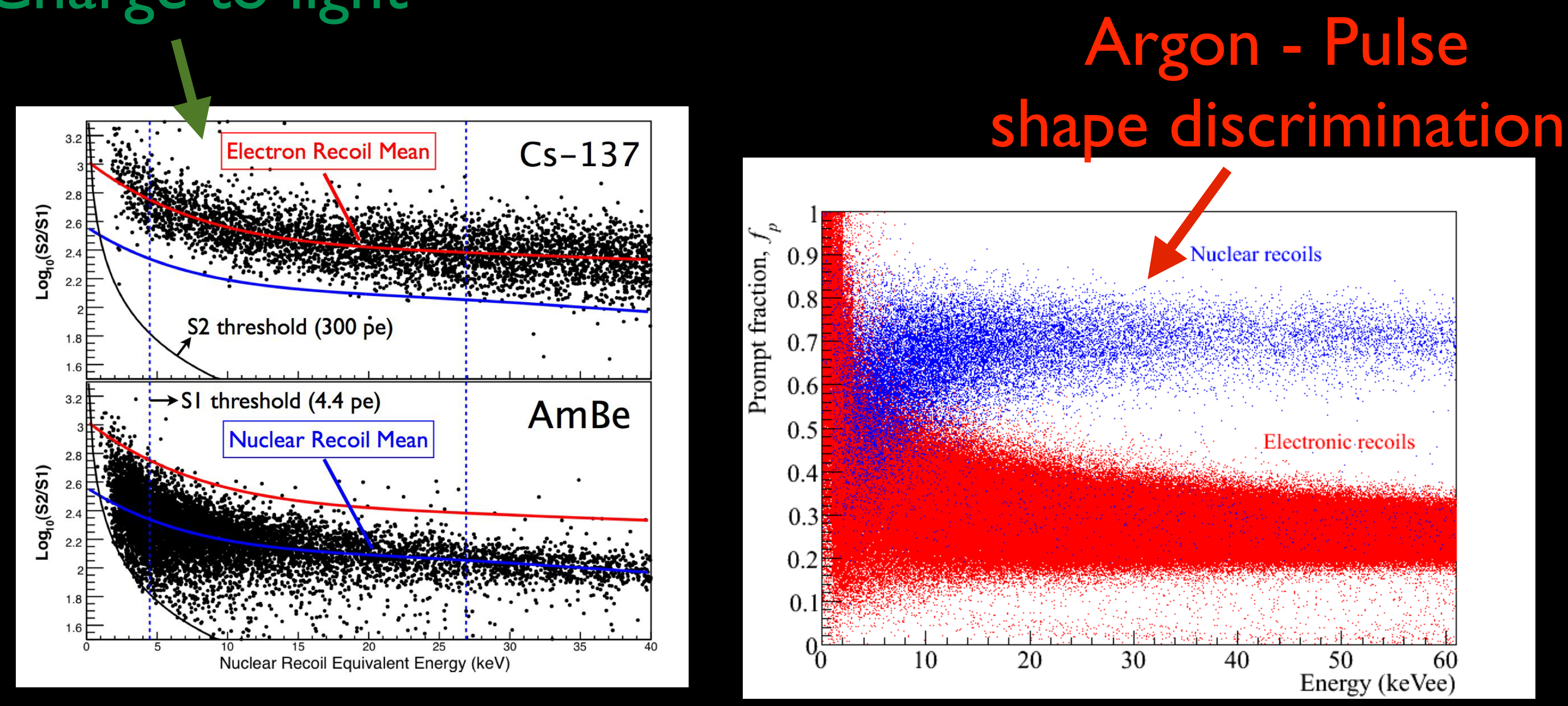


\section{Why bubble chambers?}

- By choosing superheat parameters appropriately (temperature and pressure), bubble chambers are blind to electronic recoils ( $10^{-10}$ or better)

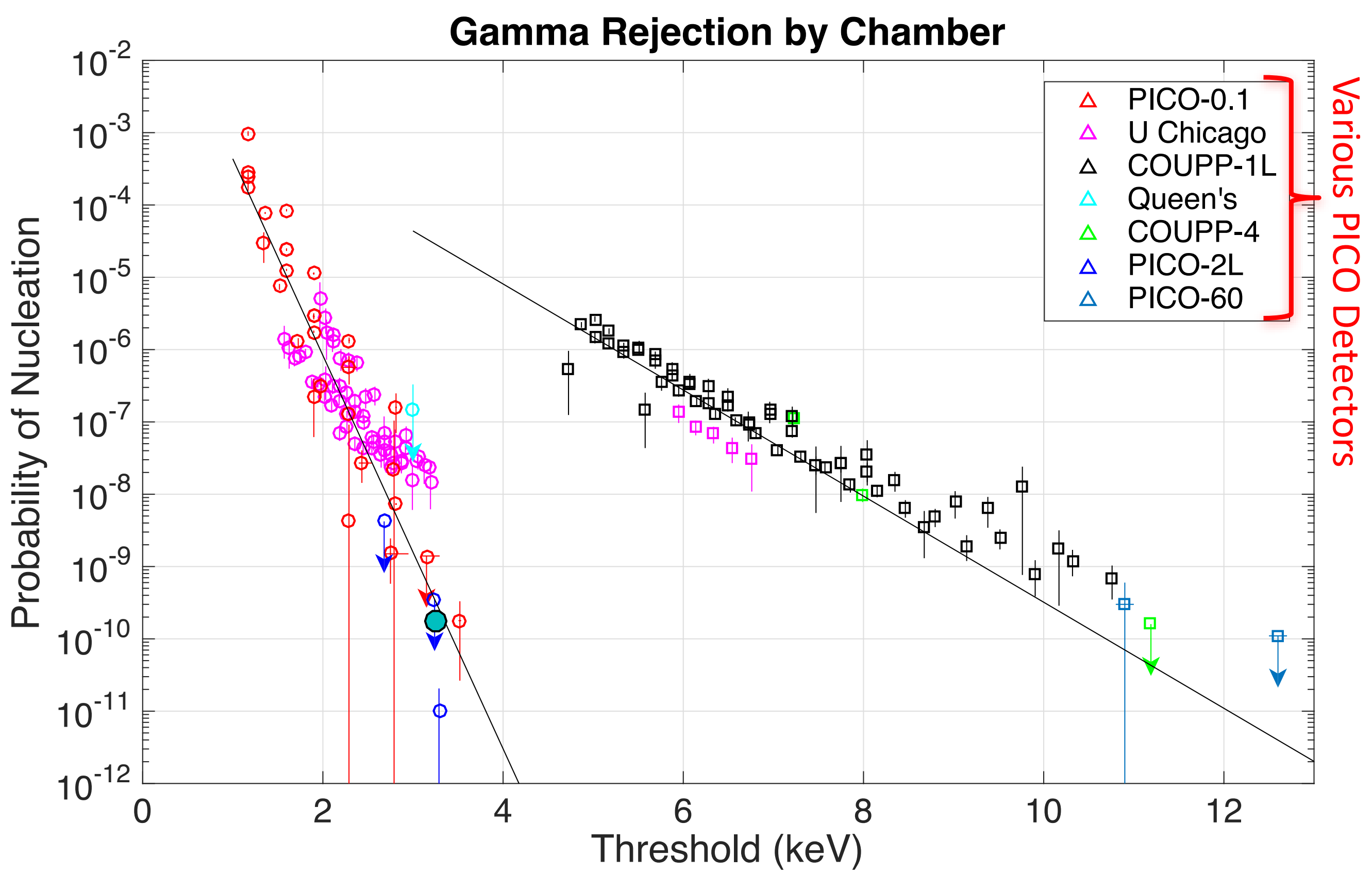




\section{Why bubble chambers?}

- By choosing superheat parameters appropriately (temperature and pressure), bubble chambers are blind to electronic recoils ( $10^{-10}$ or better)

- To form a bubble requires two things

- Enough energy

- Enough energy density - length scale must be comparable to the critical bubble size

- Electronic recoils never cross the second threshold! 


\section{Why bubble chambers?}

- Easy to identify multiple scattering events backgrounds

- Direct measurement of neutron backgrounds by measuring multiple scatters 


\section{Why bubble chambers?}

- Easy DAQ and analysis chain

- Cameras

- Piezos

- No PMTs, no cryogenics

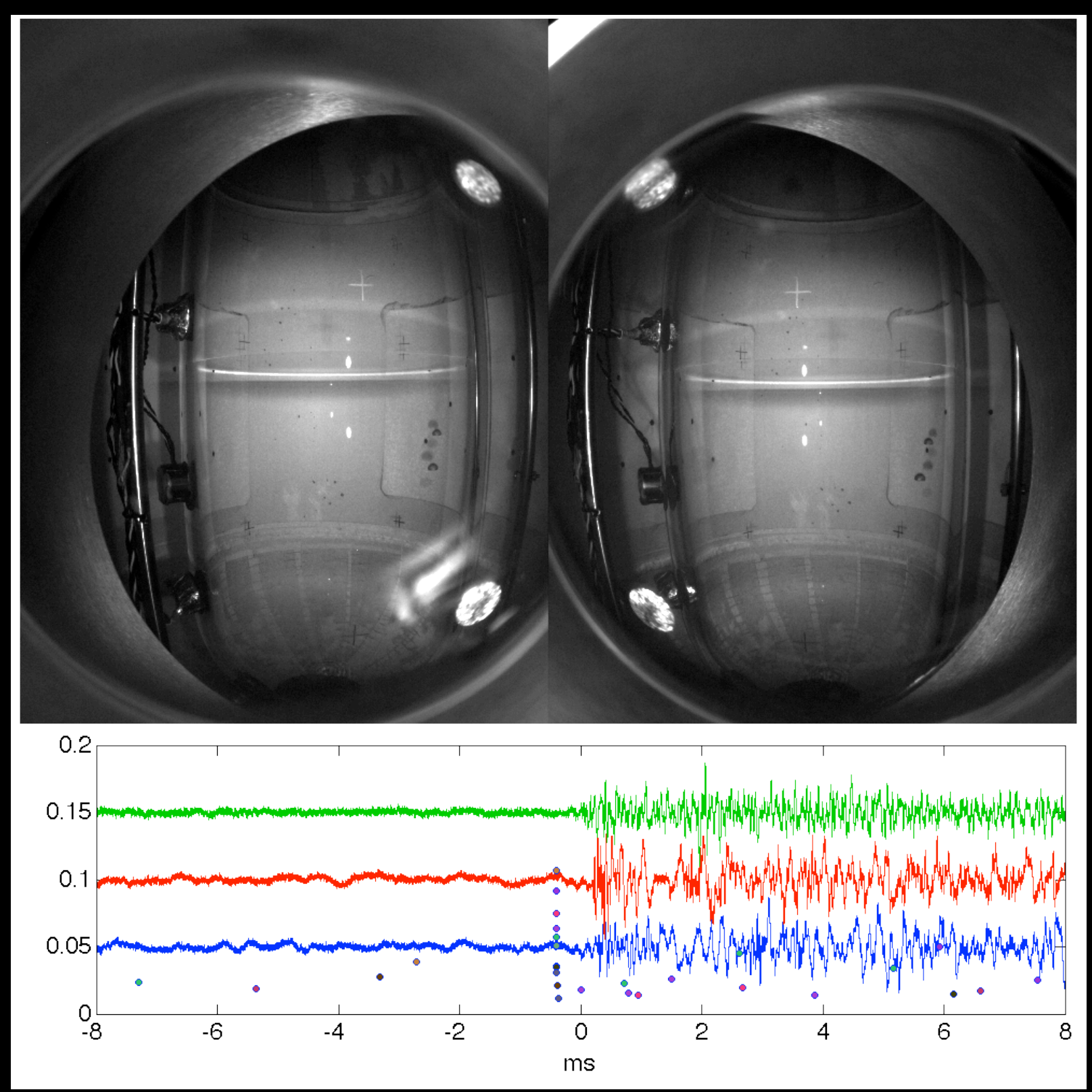




\section{Why bubble chambers?}

- Fluorine gives unique sensitivity to spin dependent proton couplings

- (Pseudo) Interchangeable targets in same detector can pin down dark matter characteristics

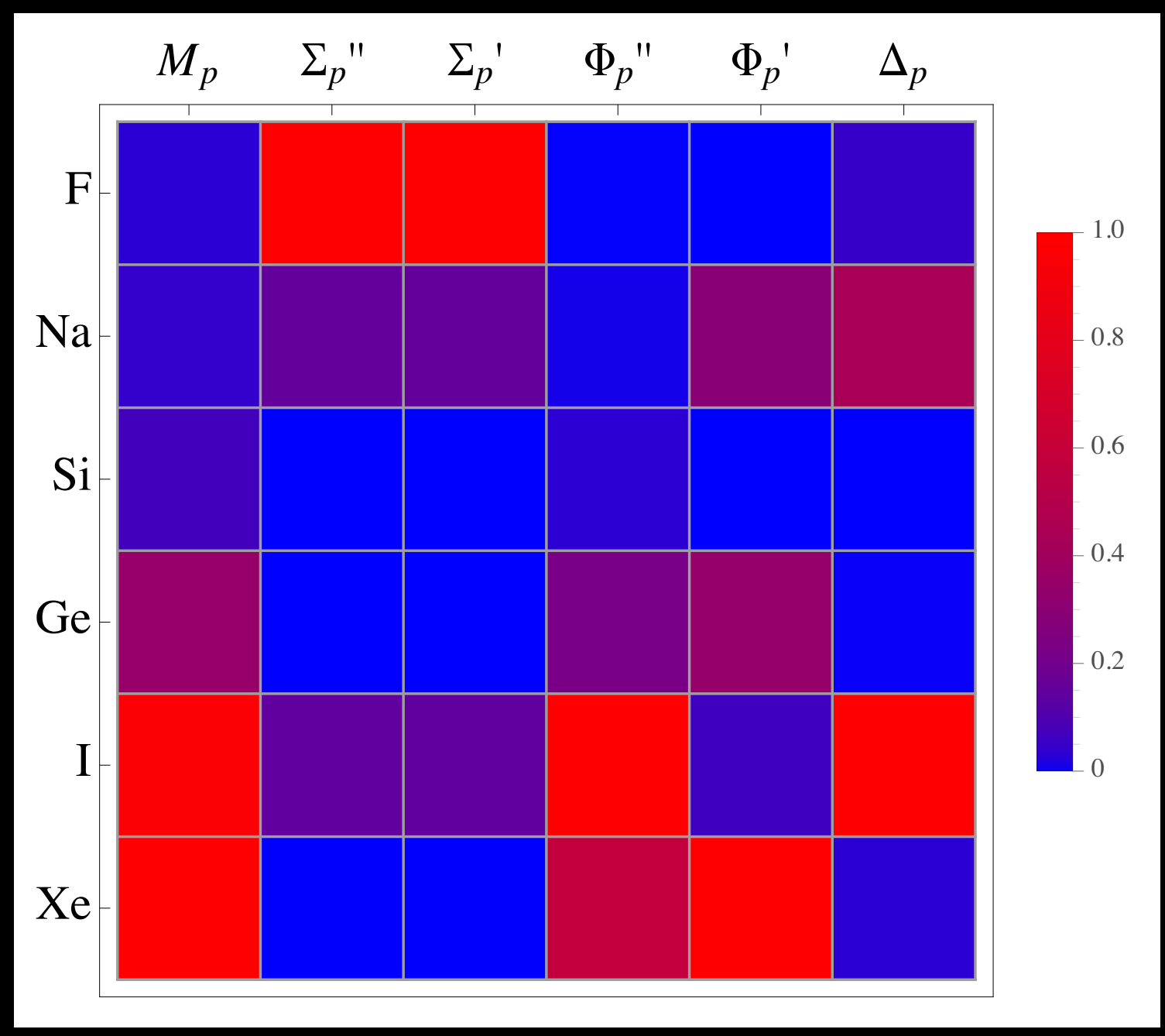

Sensitivity of different p-coupling operators to various nuclear targets

(from L. Fitzpatrick at INT Workshop, 20I4) 


\section{Why not bubble chambers?}

- Threshold detectors - no energy resolution

- Harder to distinguish some backgrounds, less information about any potential signal

- Energy threshold calibrations are hard and important

- Bubble chambers are slow - 60 s of deadtime for every event

- Overall rate must be low 


\section{Acoustic discrimination}

- Discovery that alphas were louder than nuclear recoils by PICASSO (Aubin et al, New J. Phys 10:1030 I7, 2008), and then confirmed by COUPP

- Alphas deposit energy over tens of microns

- Nuclear recoils deposit theirs in tens of nanometers

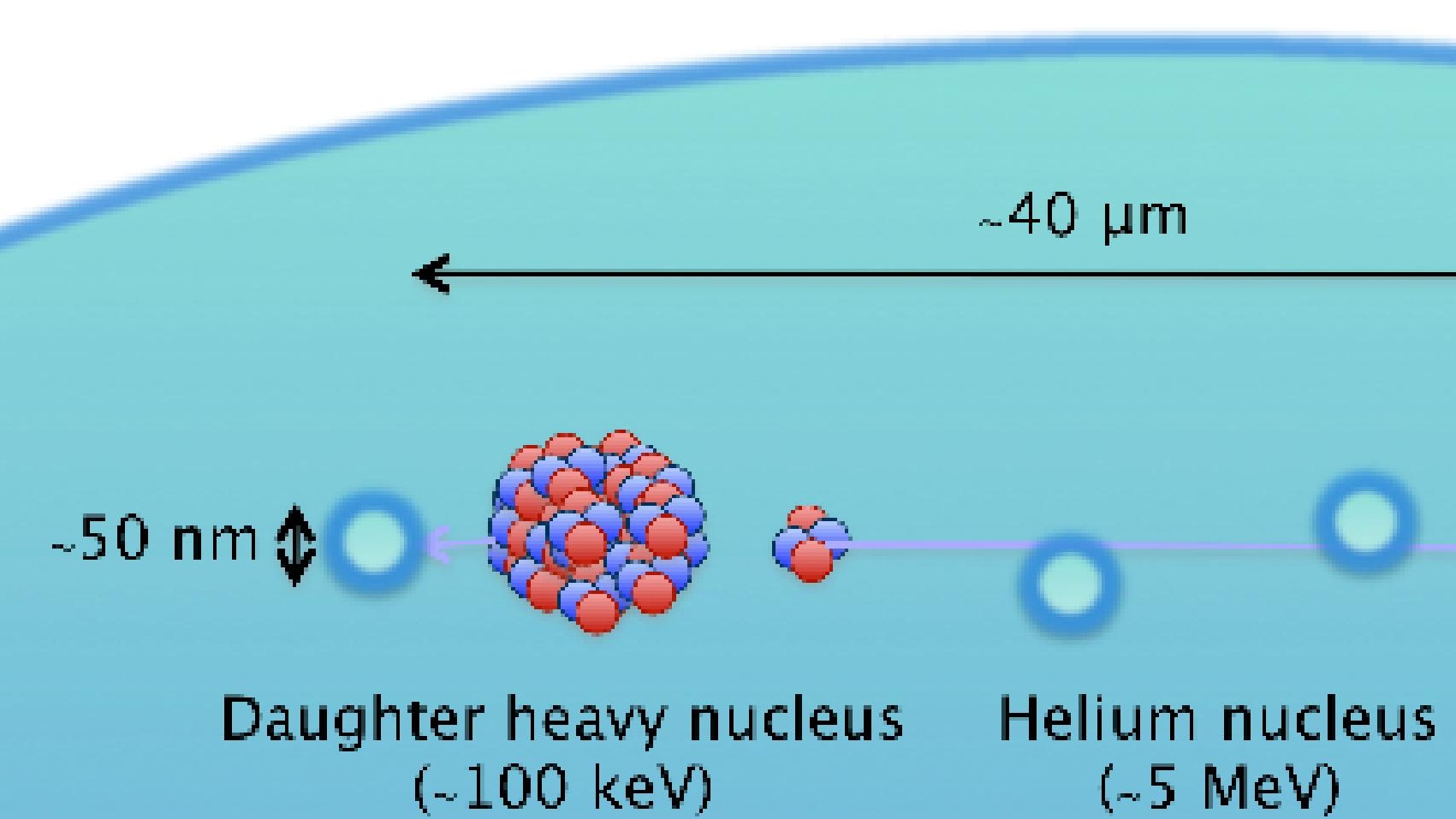

Observable bubble

$\sim \mathrm{mm}$ 


\section{Acoustic discrimination}

- Acoustic Parameter (AP) - measurement of the acoustic power of an event

- Three populations

- Neutrons (normalized to $A P=I)$

- Alphas (louder)

- Recoil-like background...

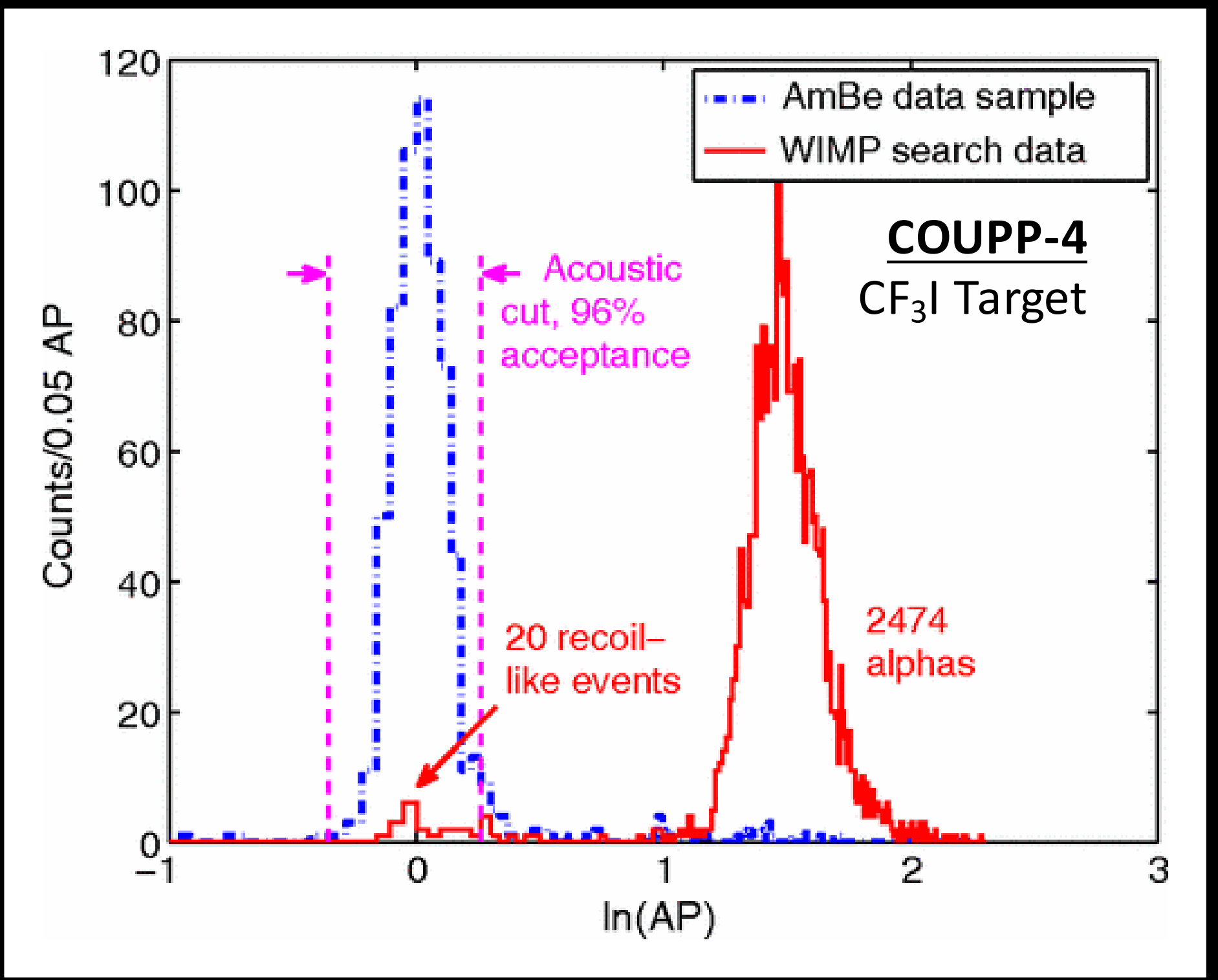




\section{Anomalous background}

- Recoil-like sounding events that otherwise do not have the characteristics of dark matter

- Spatially and temporally inhomogeneous

- PICO2L RunI

- 9 events in 32 days

- Inconsistent with known backgrounds and dark matter

- Cleaned detector of particle contamination

- PICO2L Run2

- I candidate event in 66 days

- Consistent with neutron

Run I

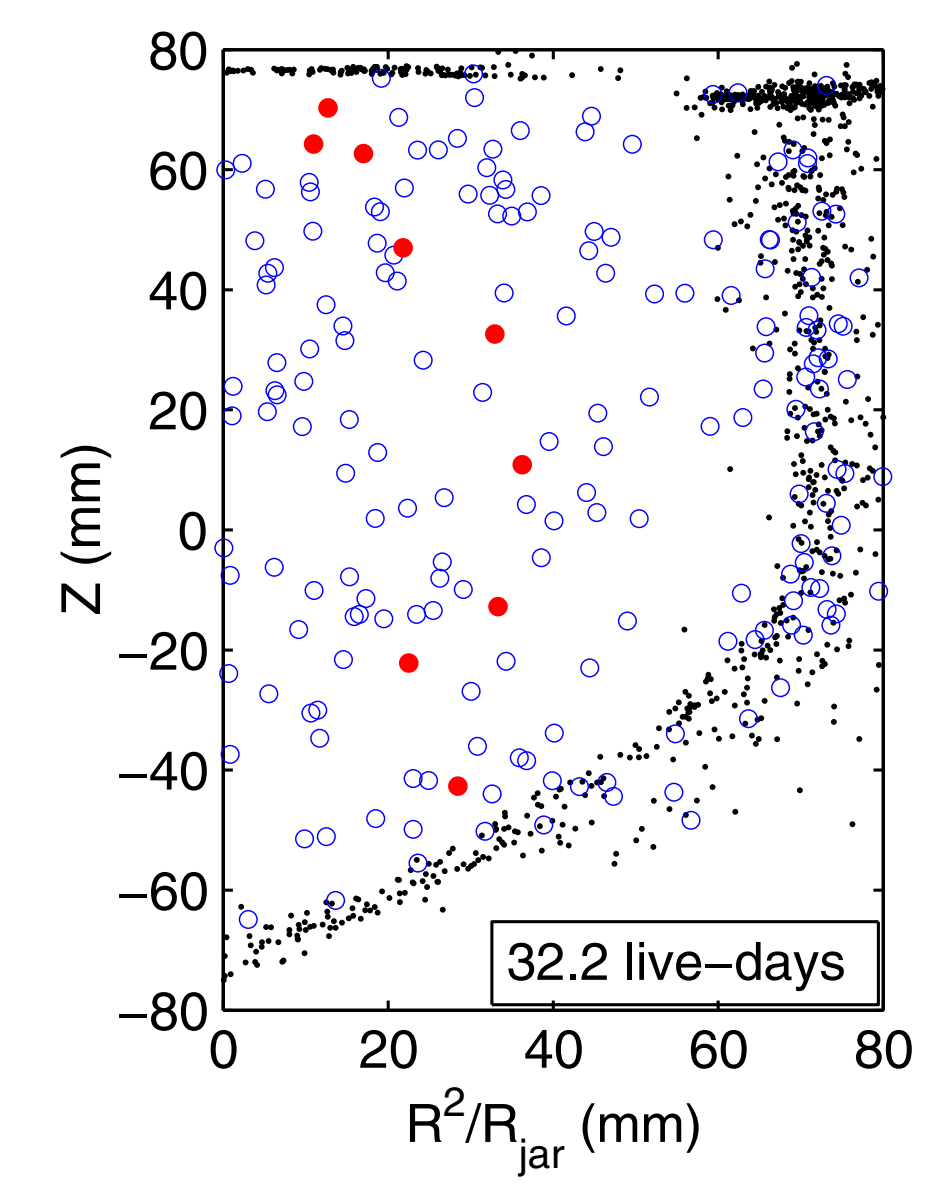

Run 2

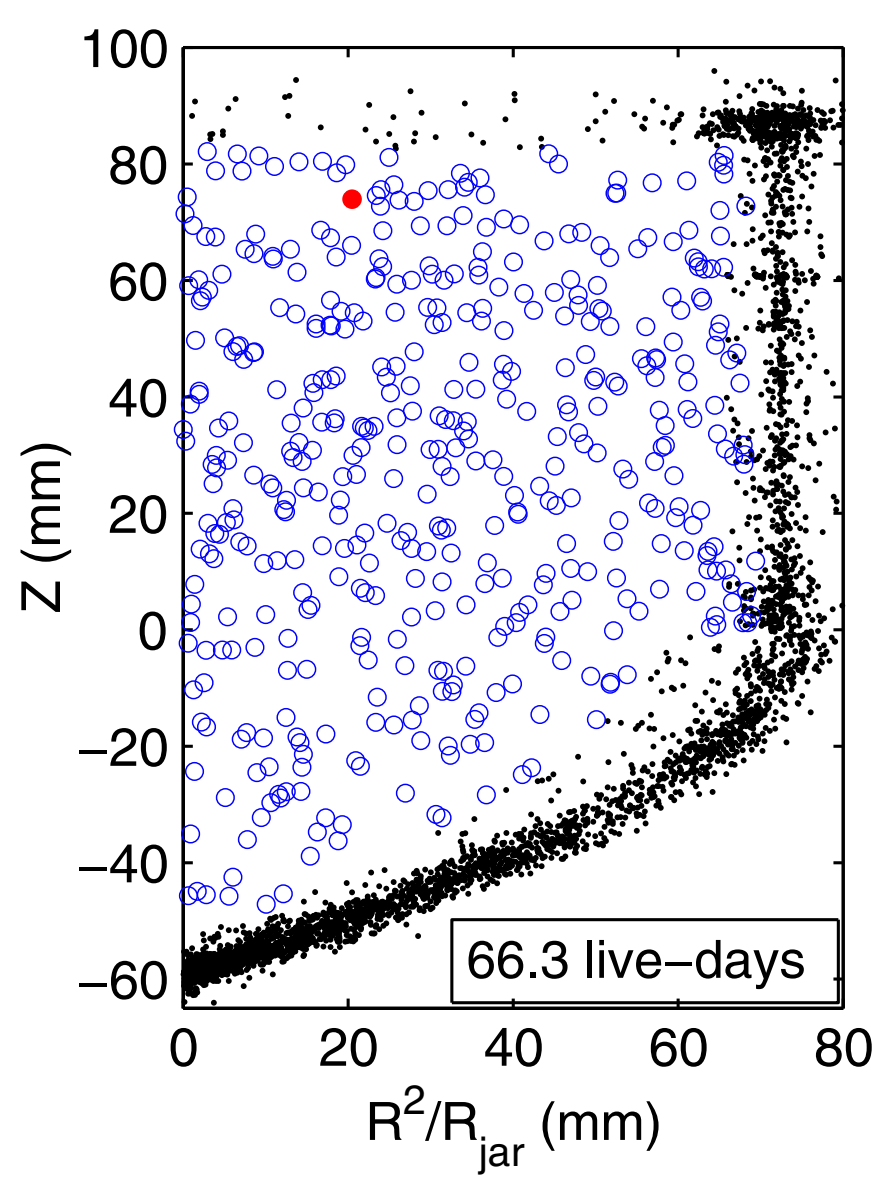
expectations 


\section{Anomalous background}

- Our hypothesis - particulate and buffer fluid (water) can lead to bubble nucleation

- Surface effects

- We need to get more serious about cleanliness
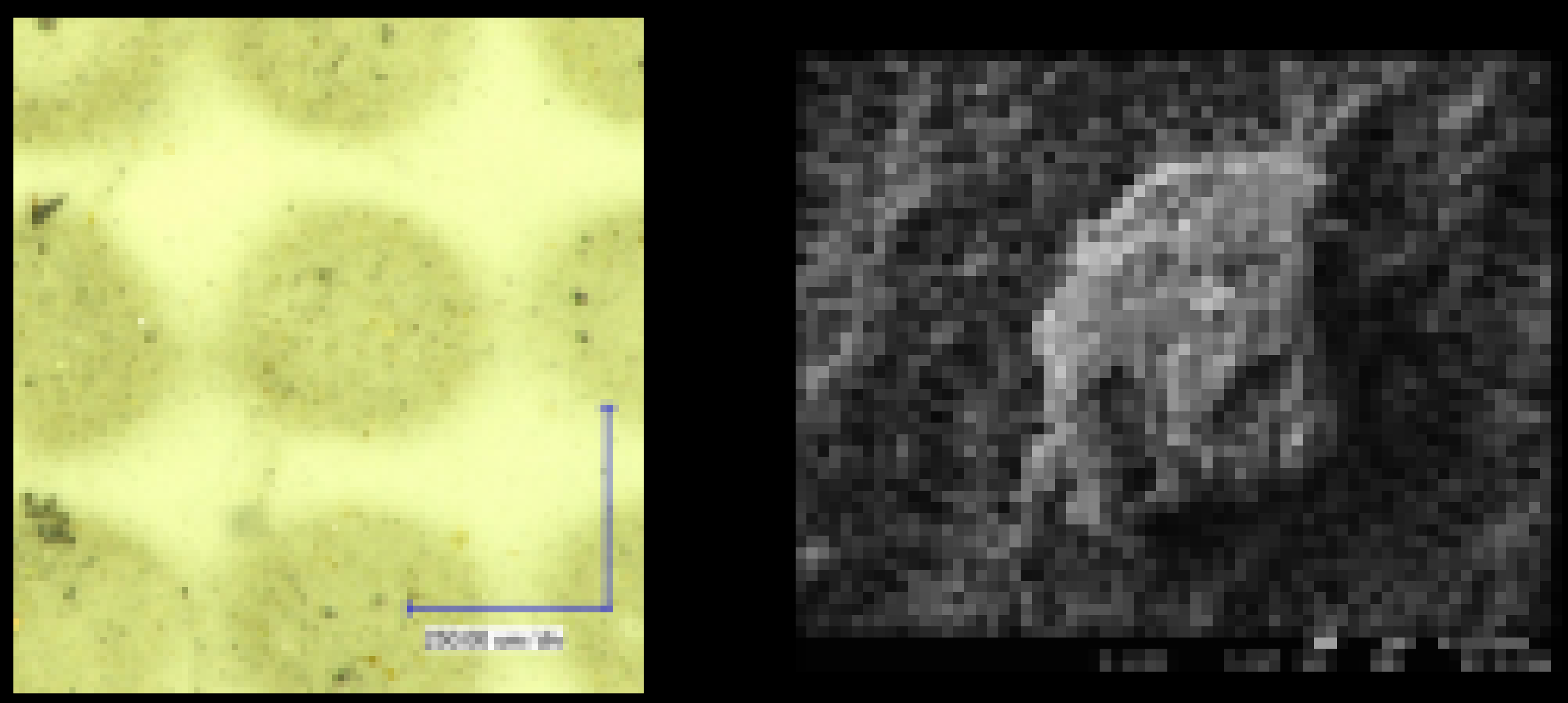


\section{PICO Timeline}

COUPP4 (20II-20I2)

CF3I 


\section{PICO Timeline}

COUPP4 (20II-20I2) CF3I

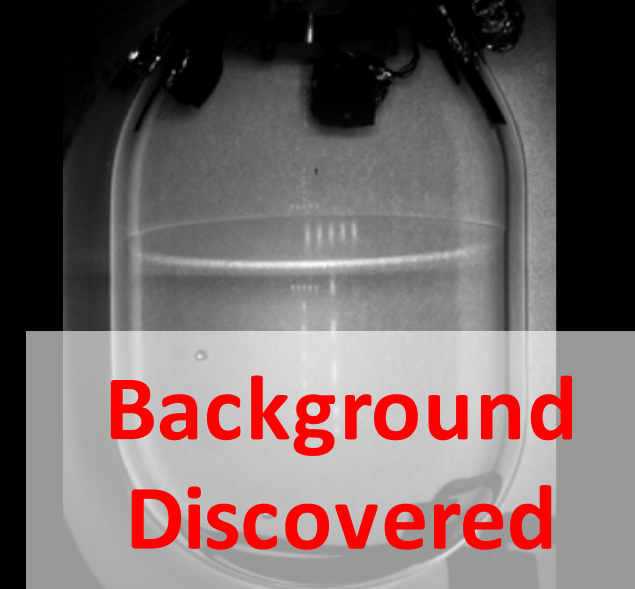


COUPP4 (20II-20I2)

CF3I

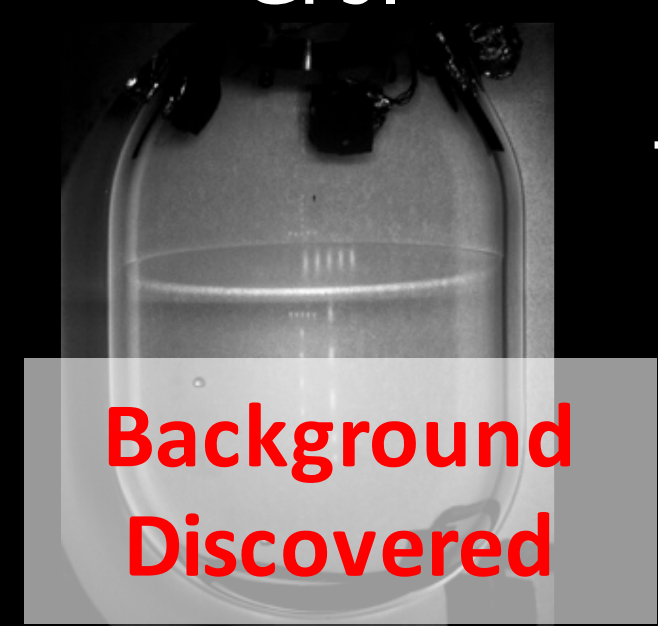

Try changing fluids

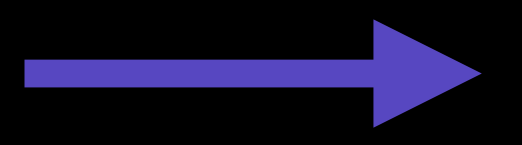

Discovered

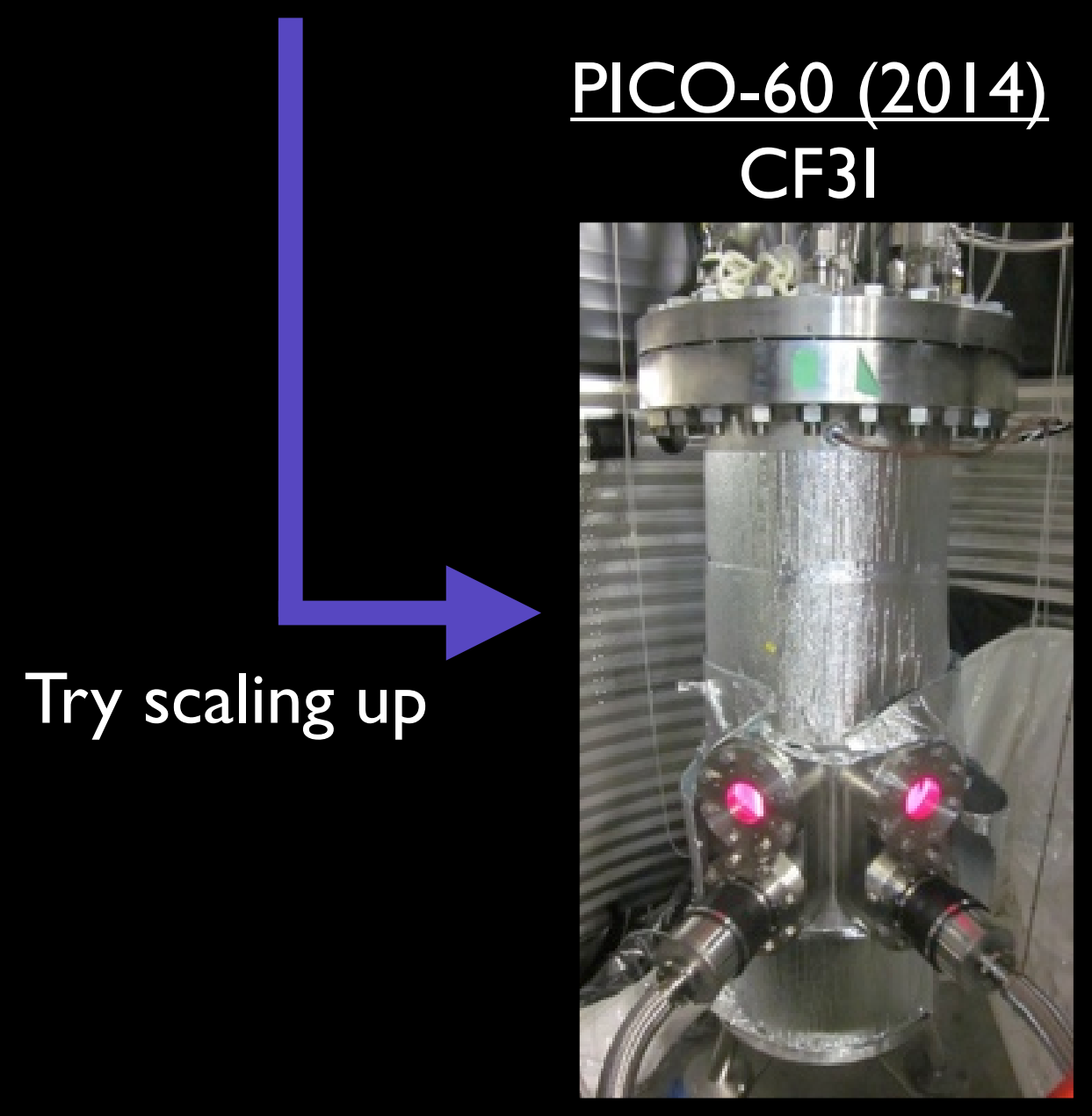

PICO-2L (2014)

C3F8

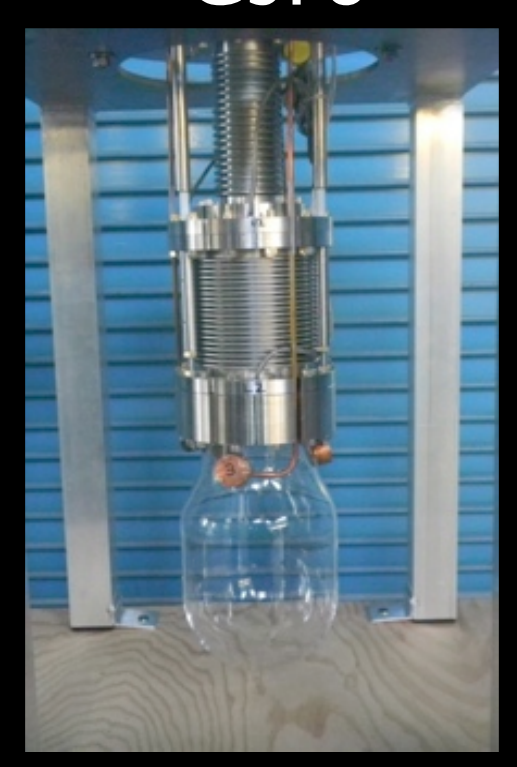




\section{PICO Timeline}

COUPP4 (20I I-20I2)

CF3I

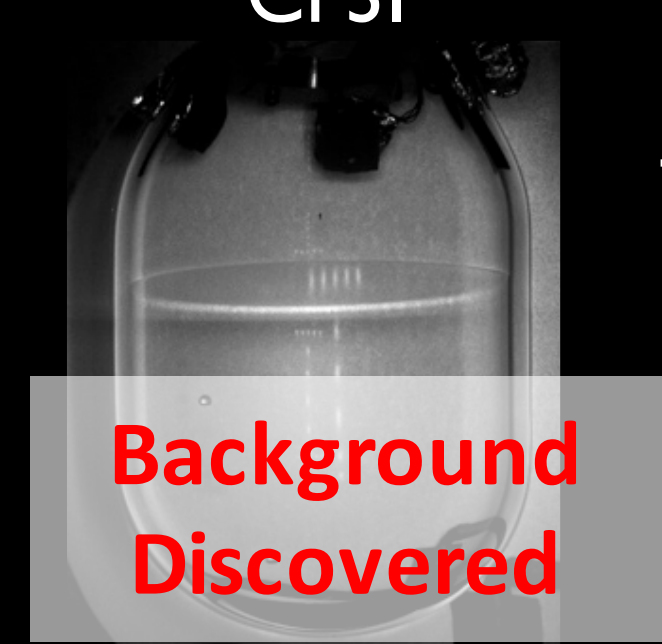

$$
\text { PICO-2L (2014) }
$$

C3F8

Try changing fluids
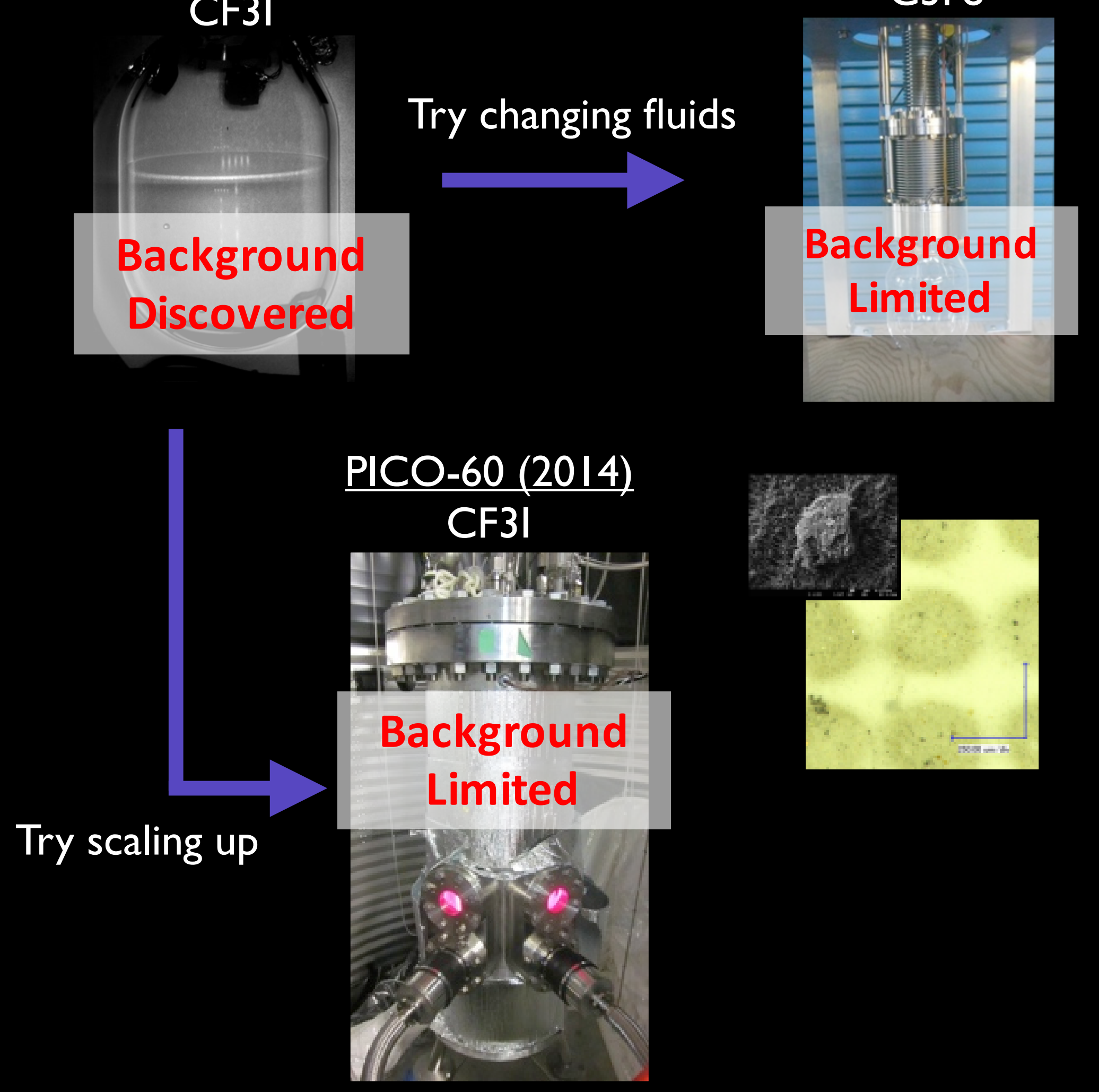


\section{PICO Timeline}

$\frac{\text { COUPP4 }(20||-20 \mid 2)}{\text { CF3I }}$

CF3I

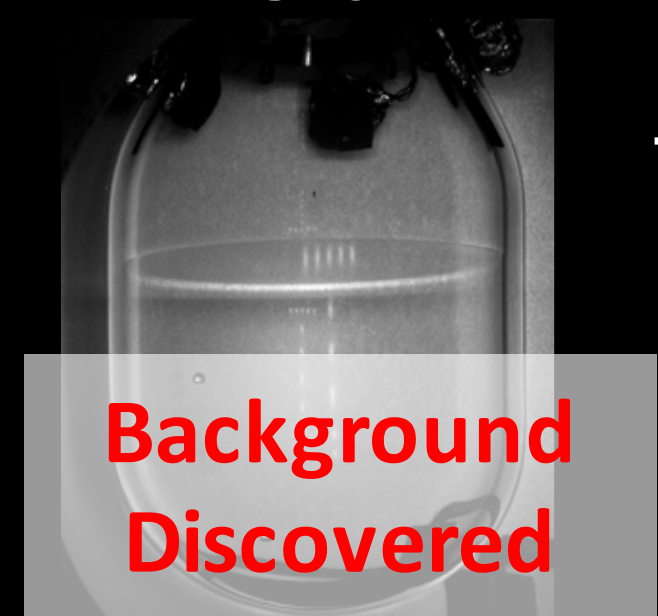

Try changing fluids

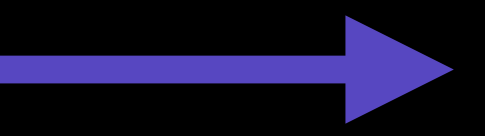

Background
PICO-2L (20|4)

C3F8

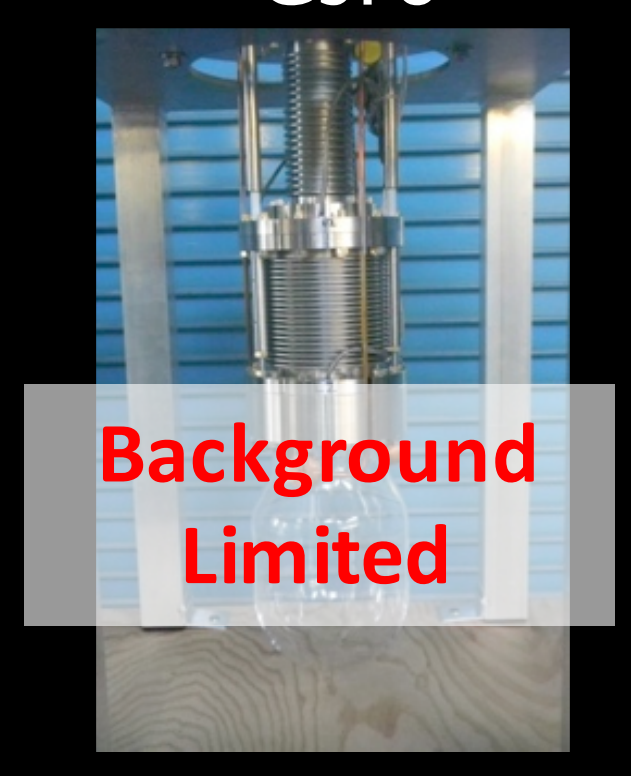

PICO-60 (20|4) CF3l

Background Limited

Try scaling up

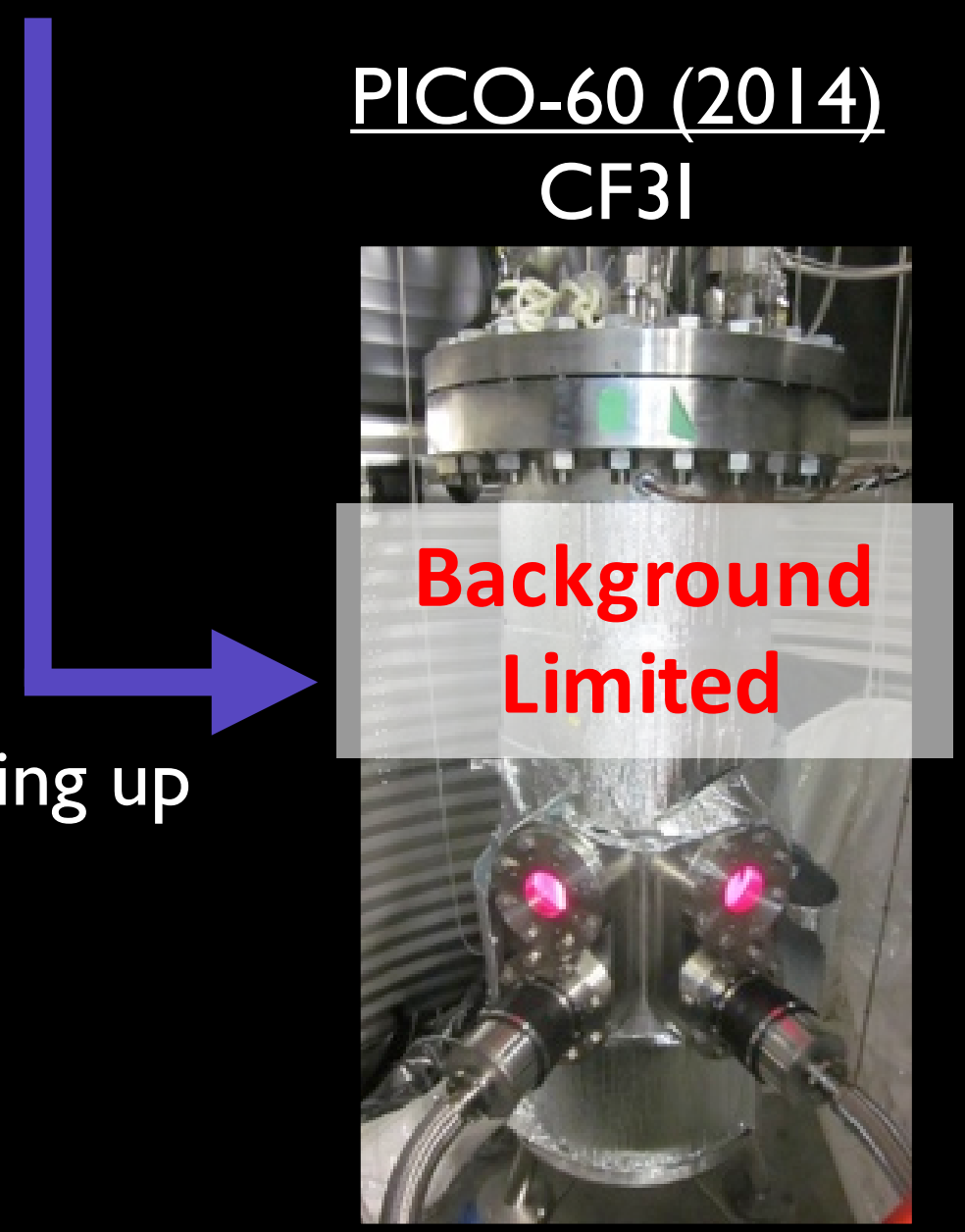

PICO-2L (2016) C3F8

Try improved cleaning

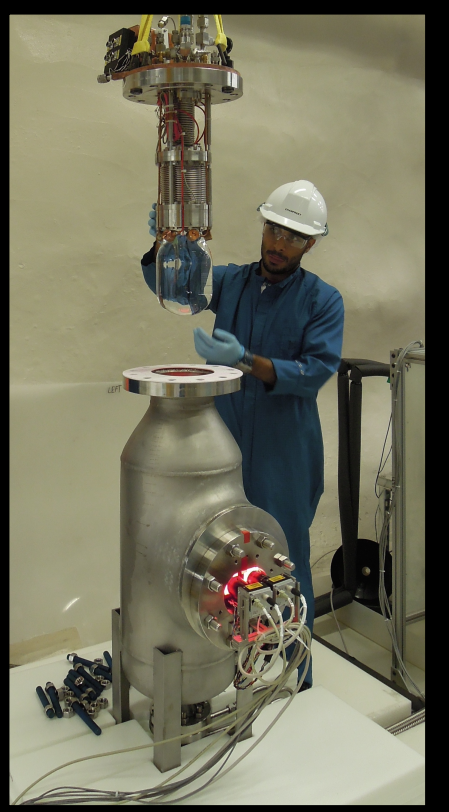




\section{PICO Timeline}
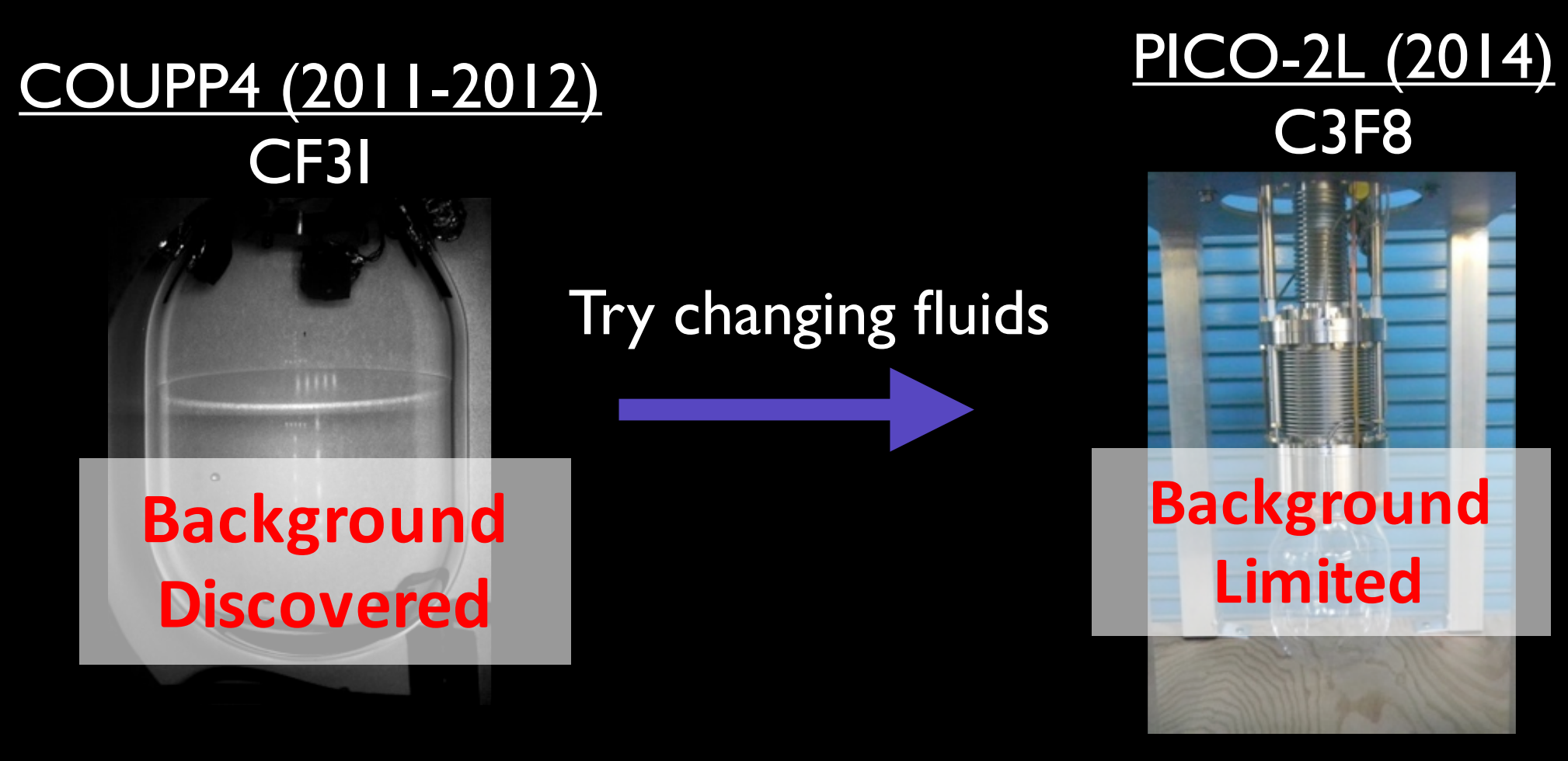

PICO-60 (20 14$)$ CF3I

\section{Background} Limited

Try scaling up

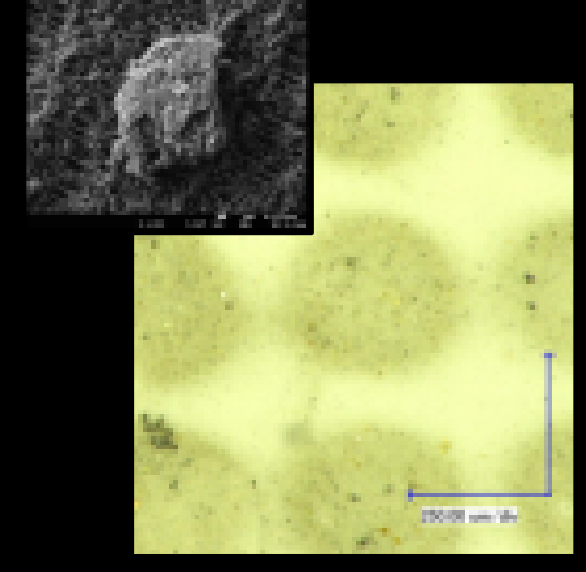

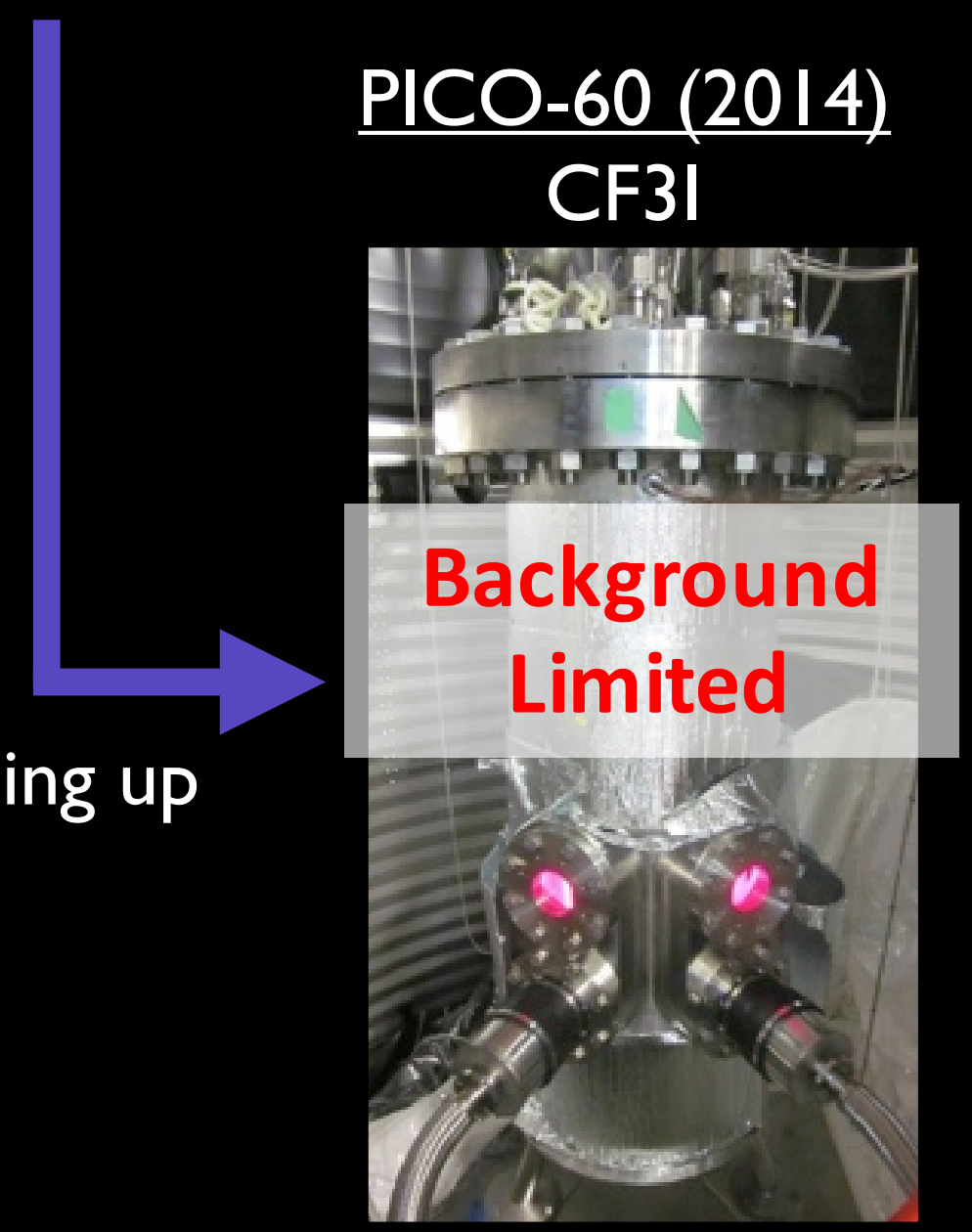

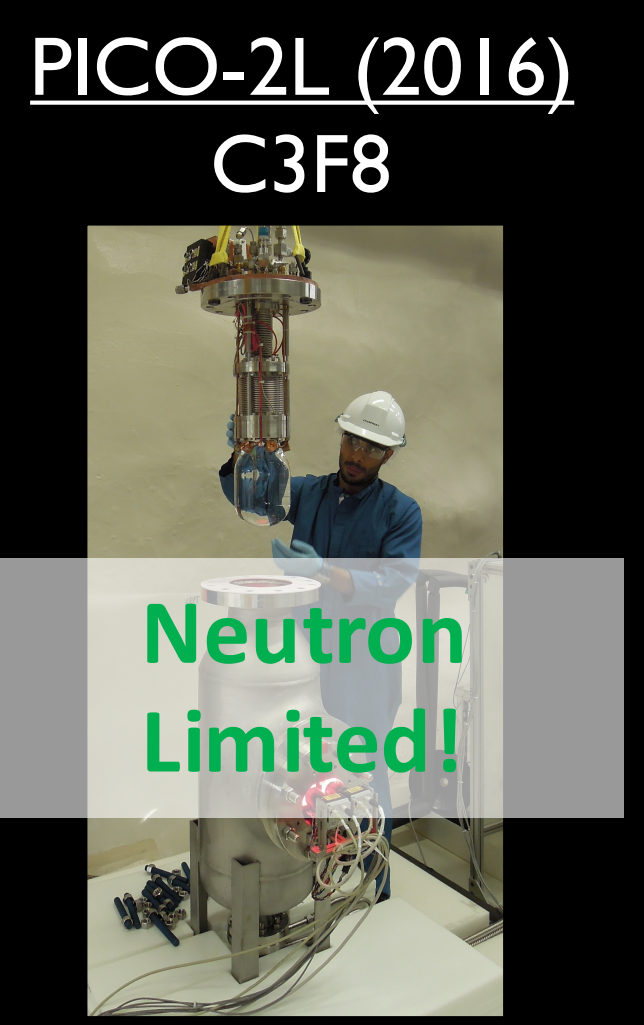

Try improved cleaning

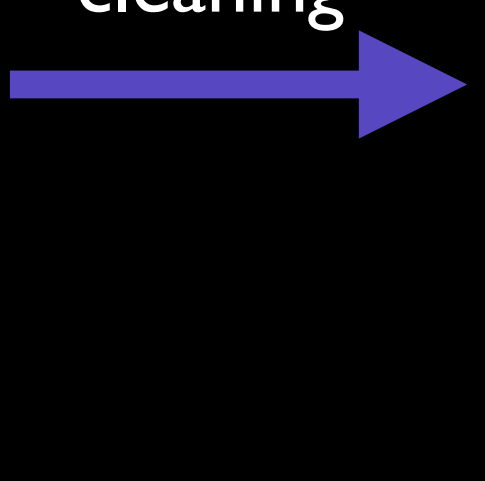




\section{PICO Timeline}
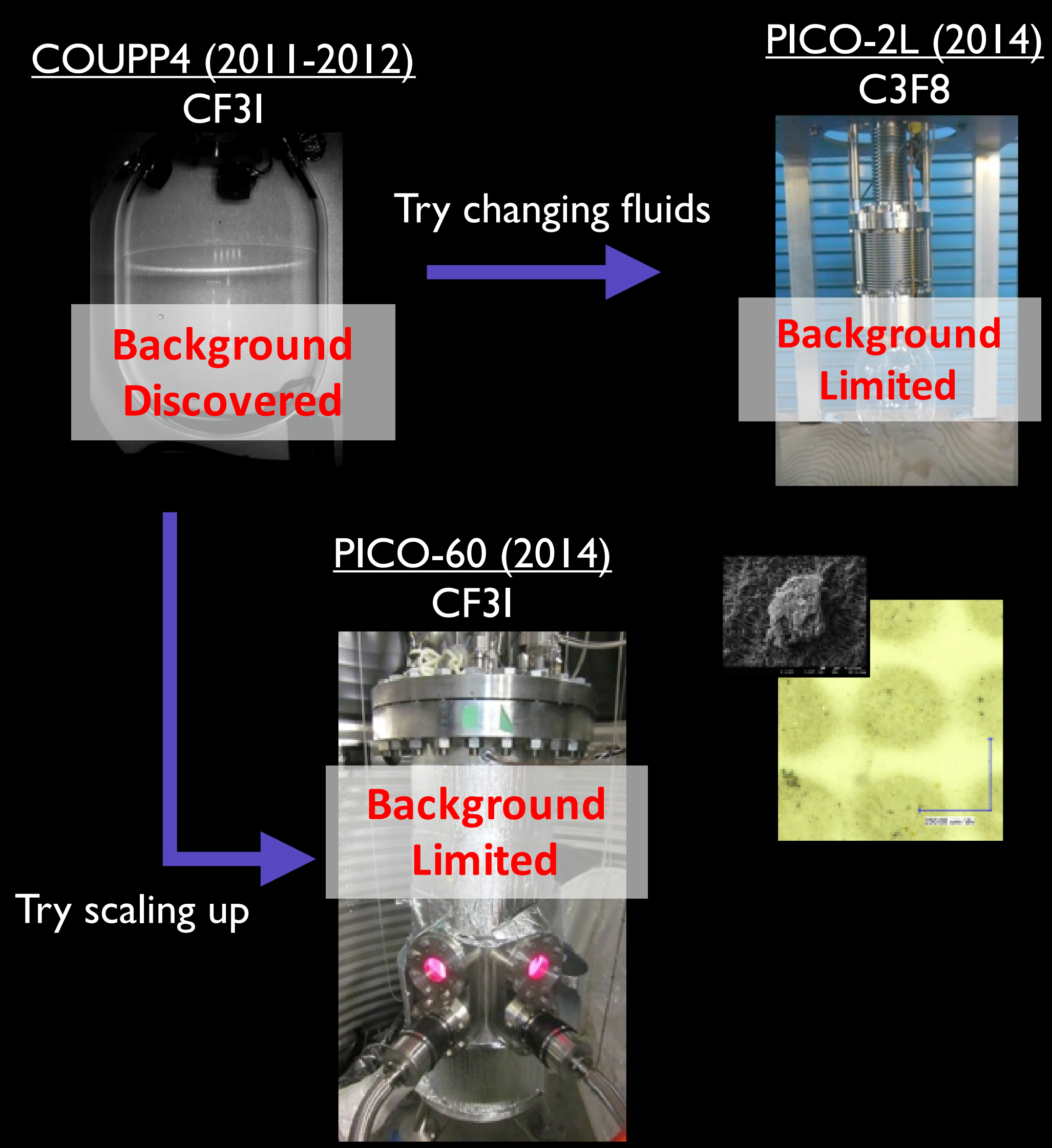
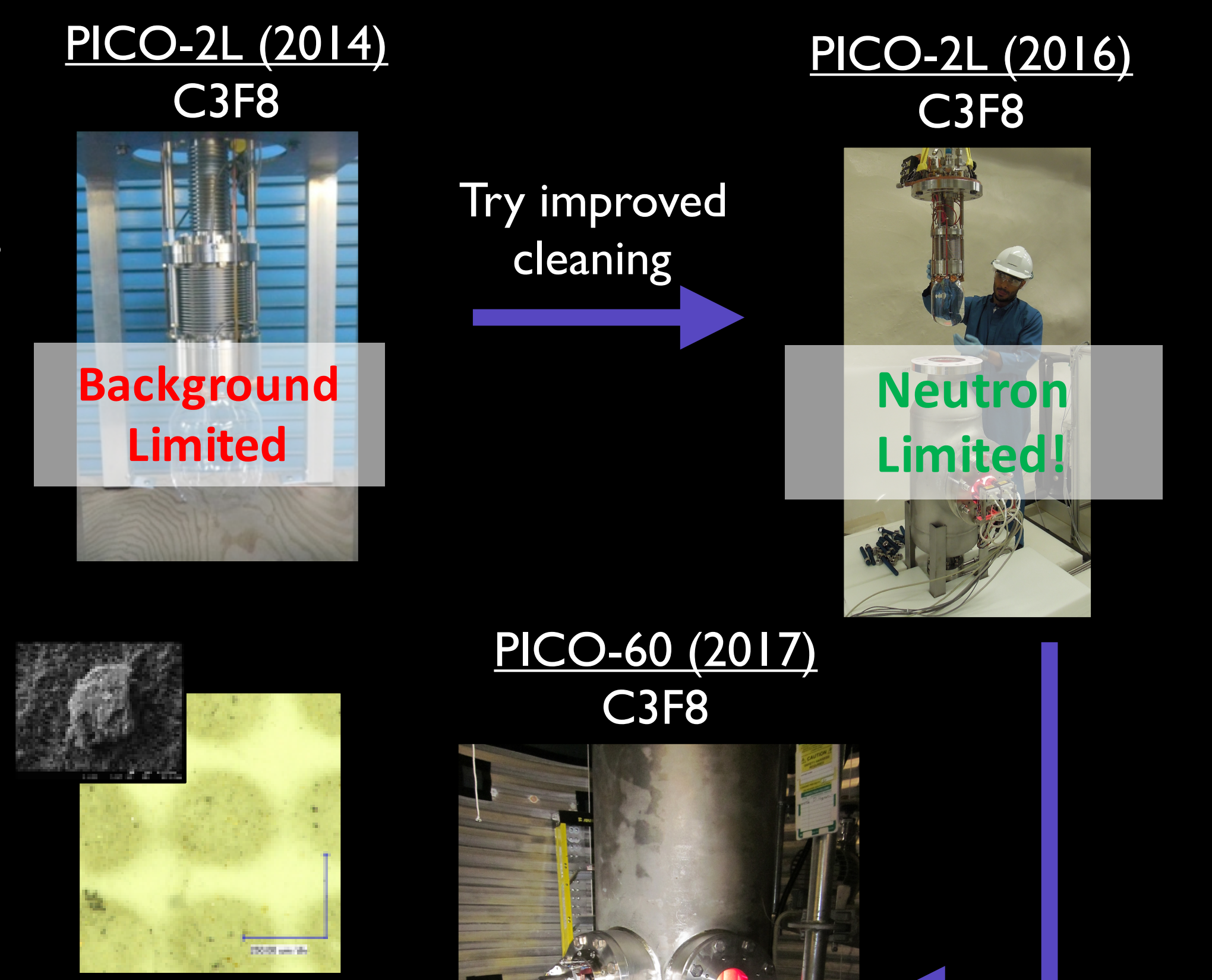

\section{PICO-60 (2017) C3F8}

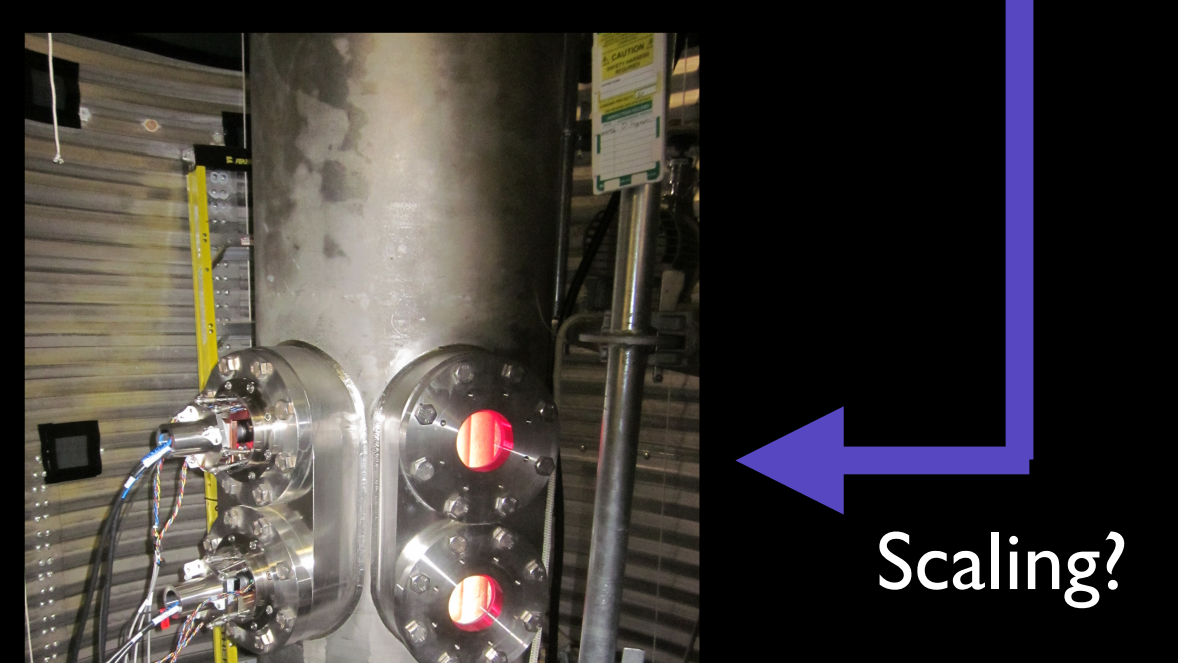




\section{PICO Timeline}
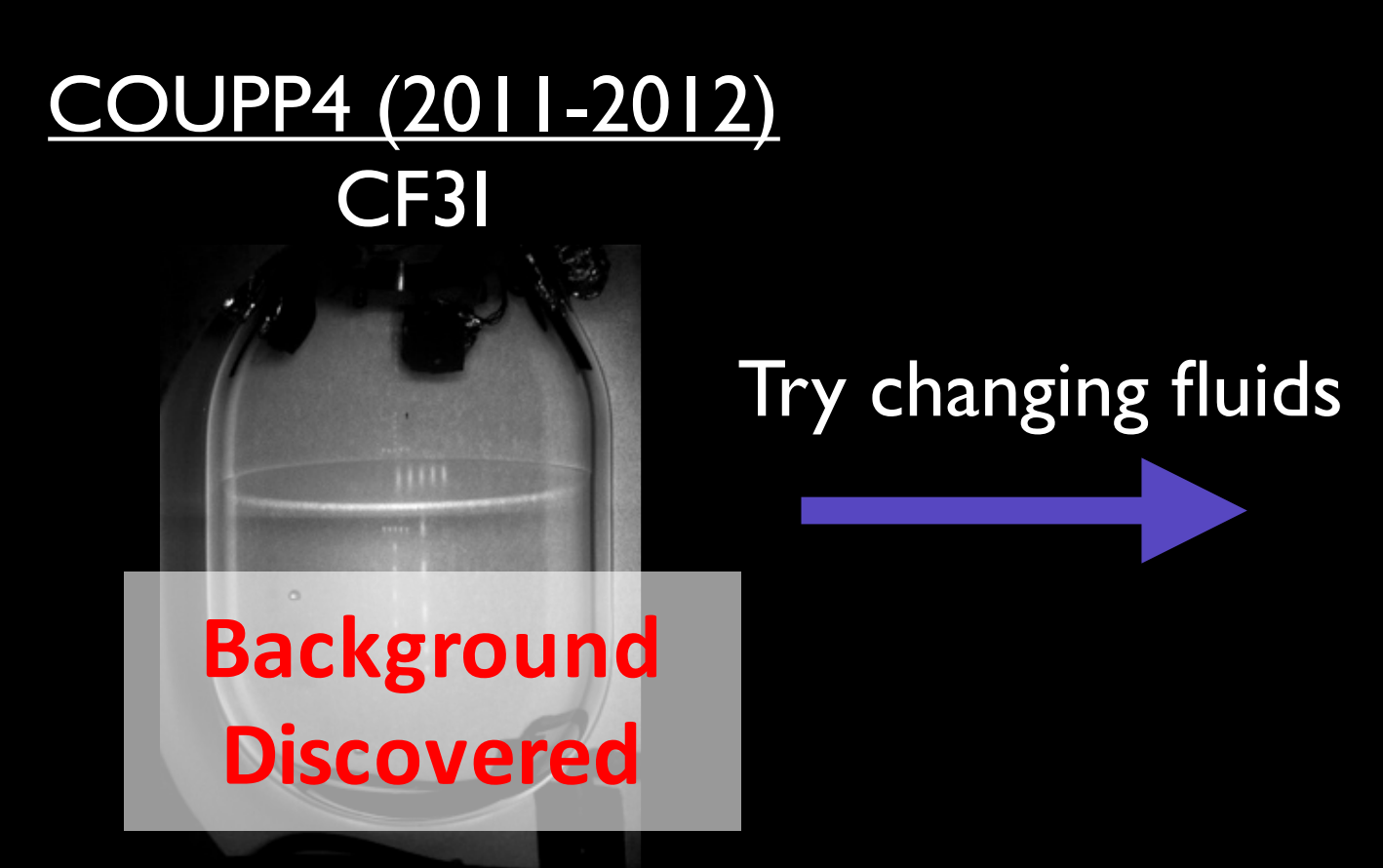

PICO-2L (2016) C3F8

Try improved cleaning

Background Limited
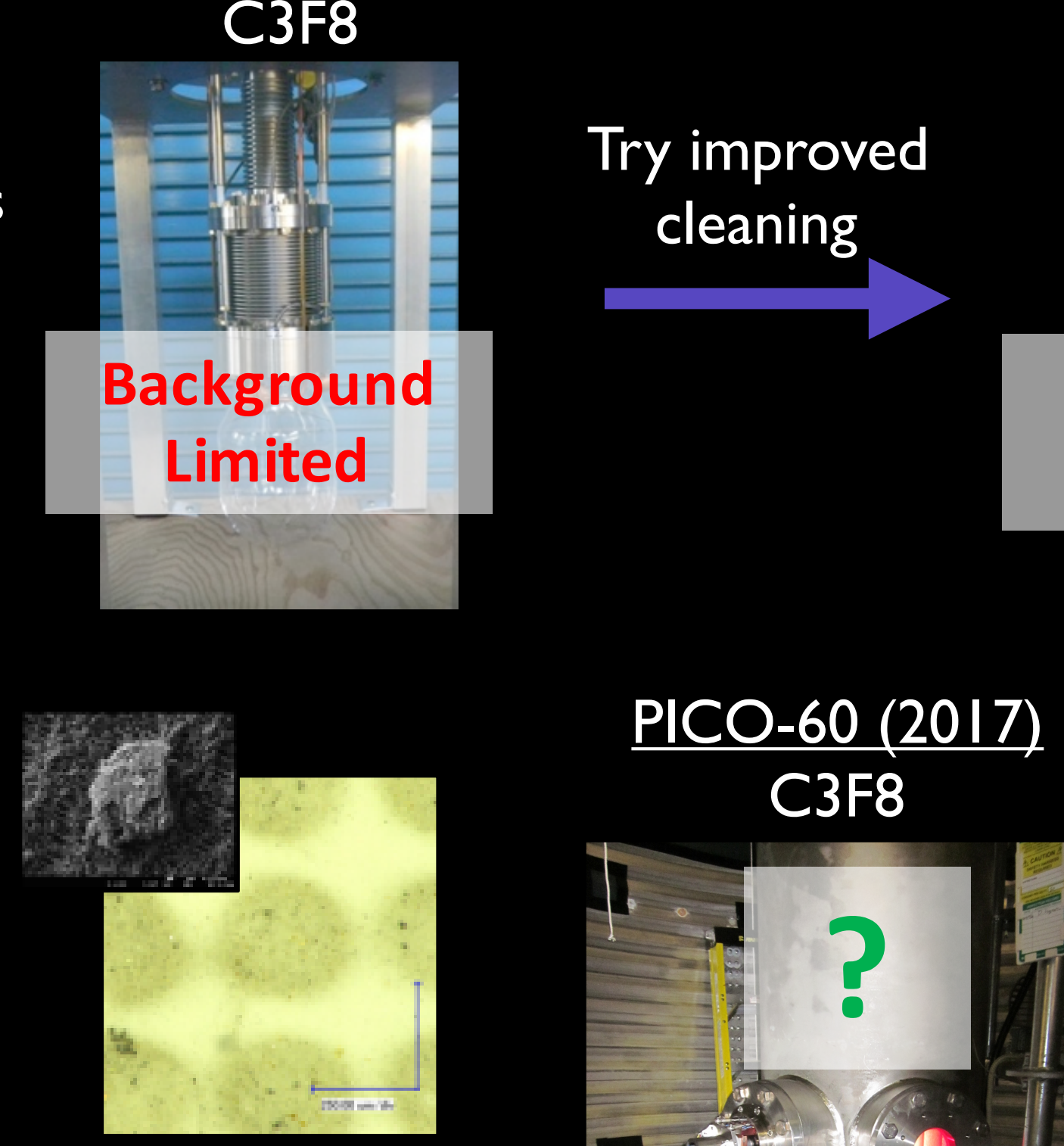

PICO-60 (2017) C3F8

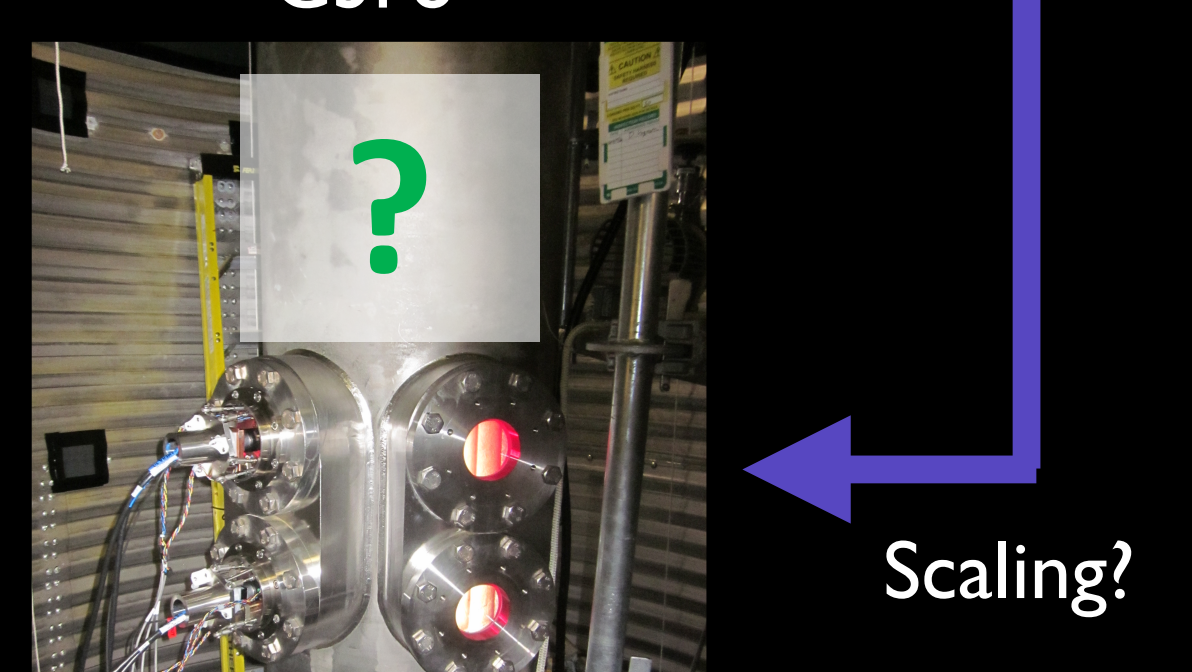

PICO-60 (2014) CF3I

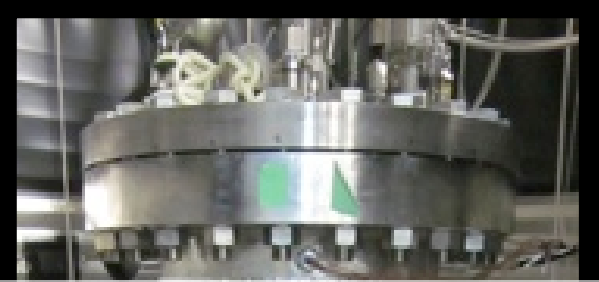

\section{Background} Limited

Try scaling up 


\section{PICO-60 (Run 2)}

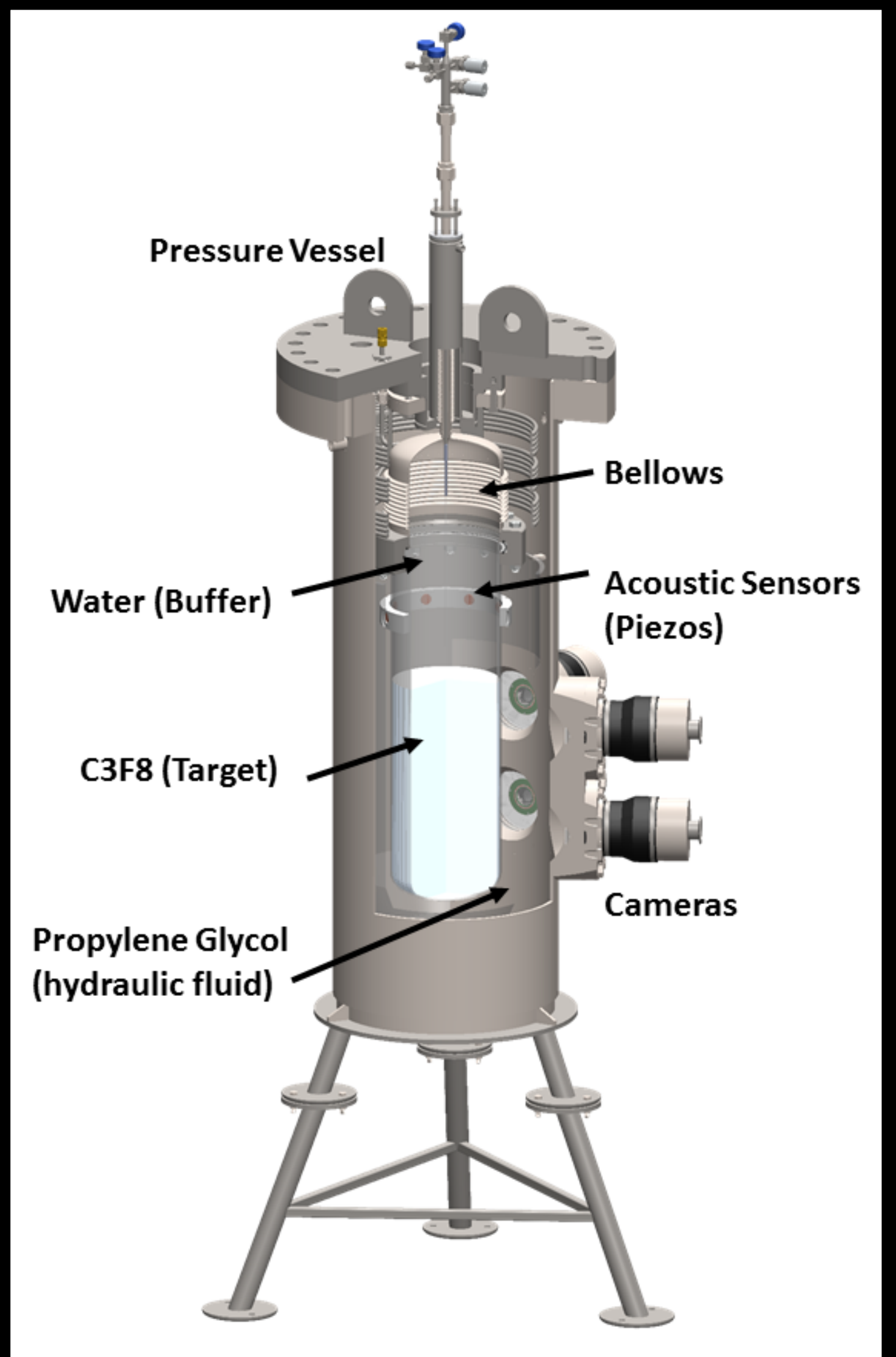

- Four cameras to observe bubbles over larger target

- Eight piezo to listen to bubble growth

- Five died early in the run

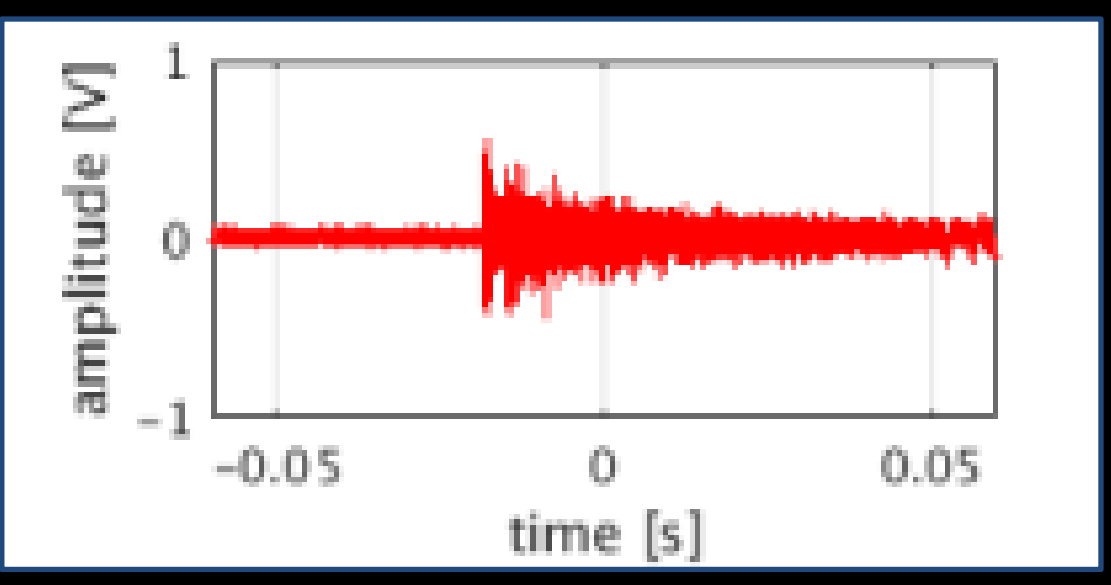




\section{PICO-60 Run 2 Cleaning}

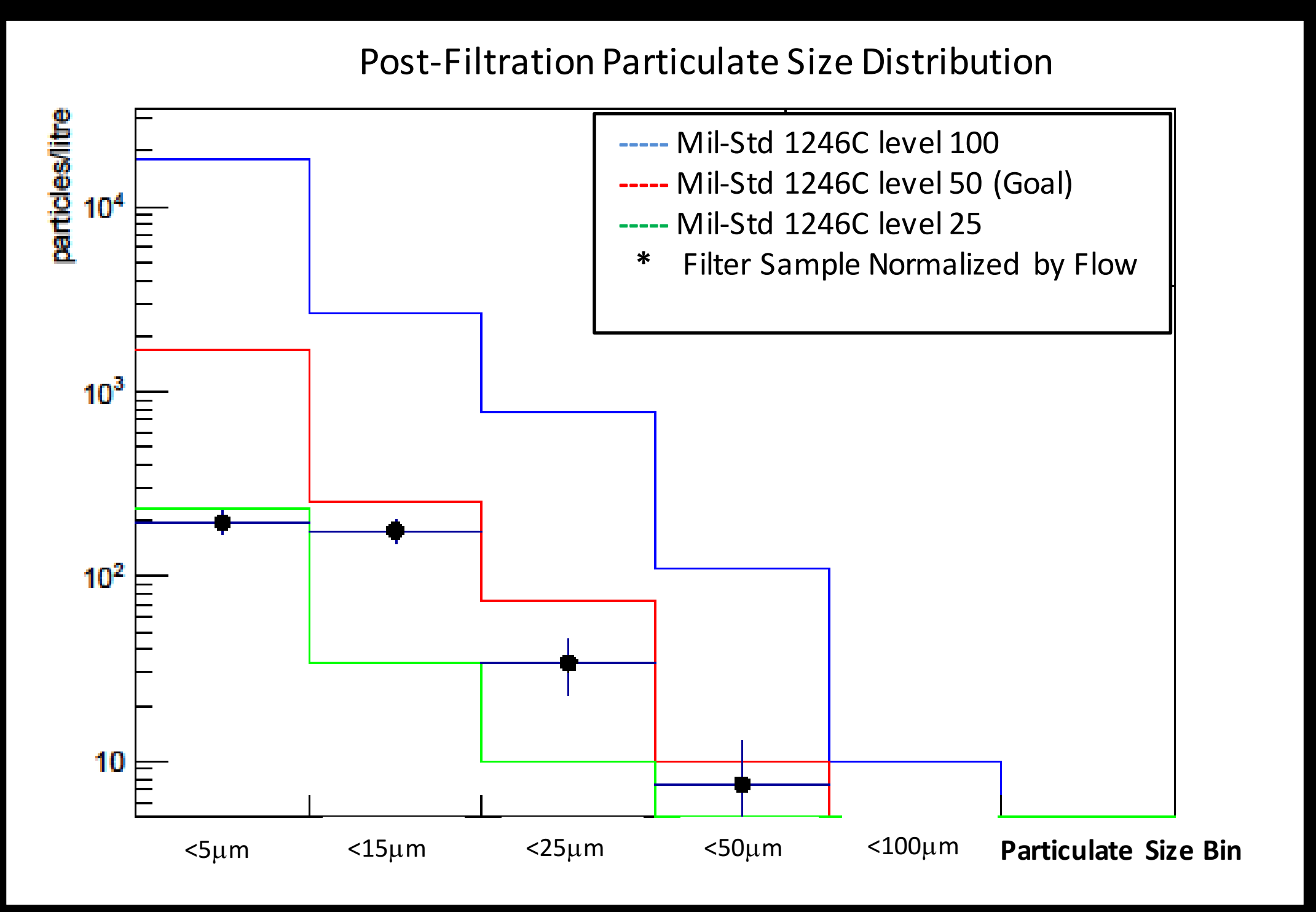

- Inner vessel and all plumbing cleaned to IEST-STD- 246 Level 50

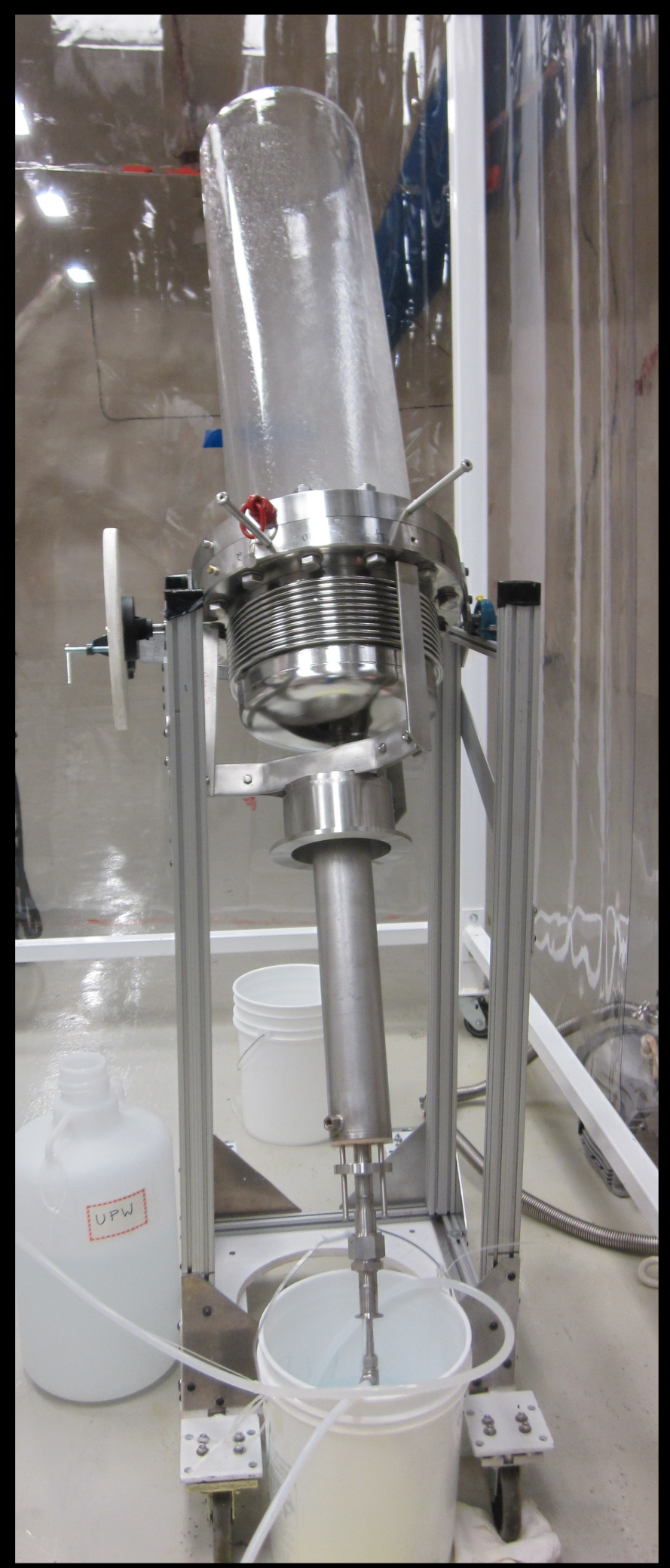




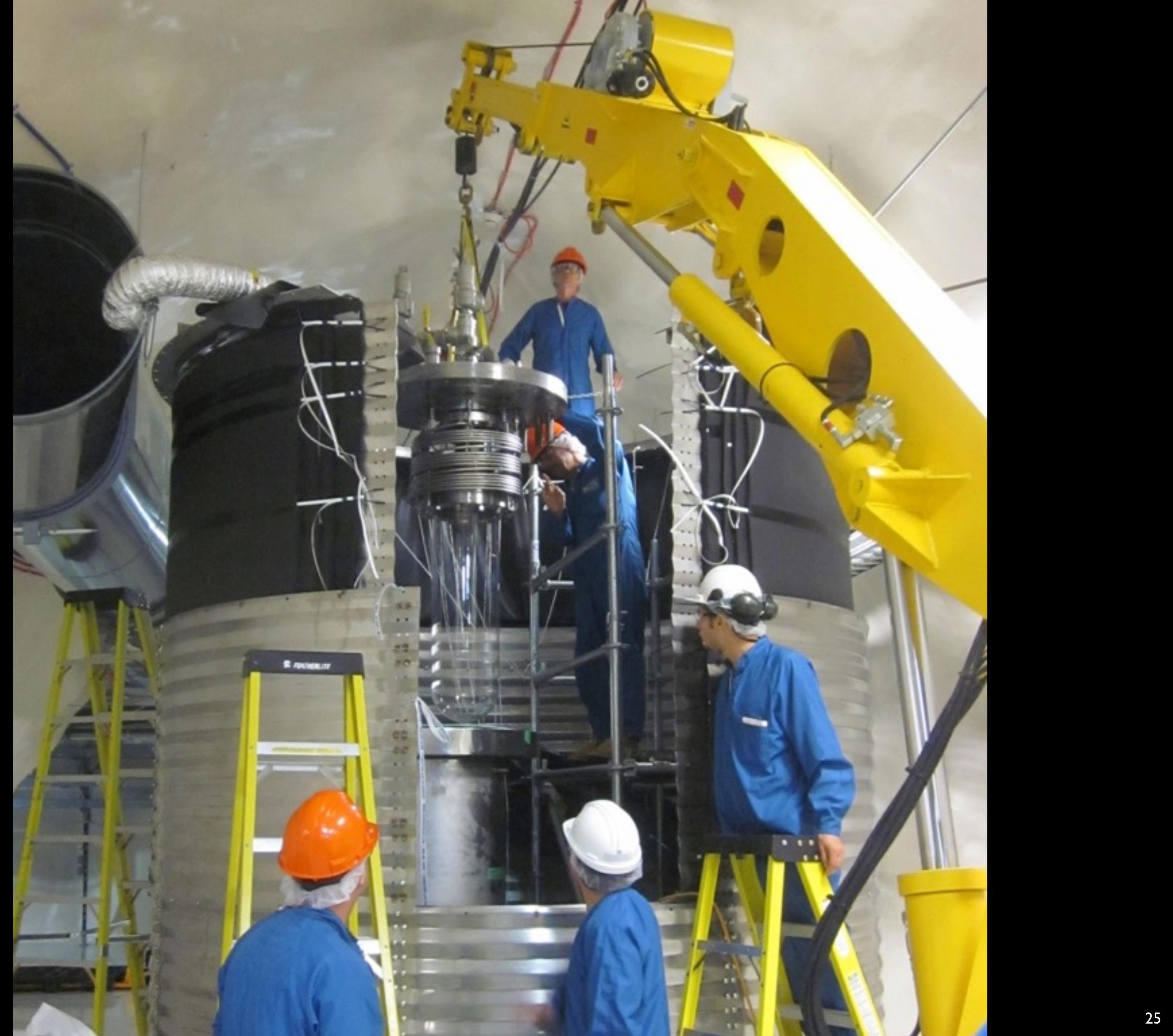




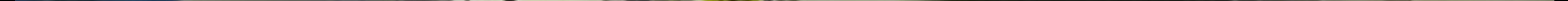



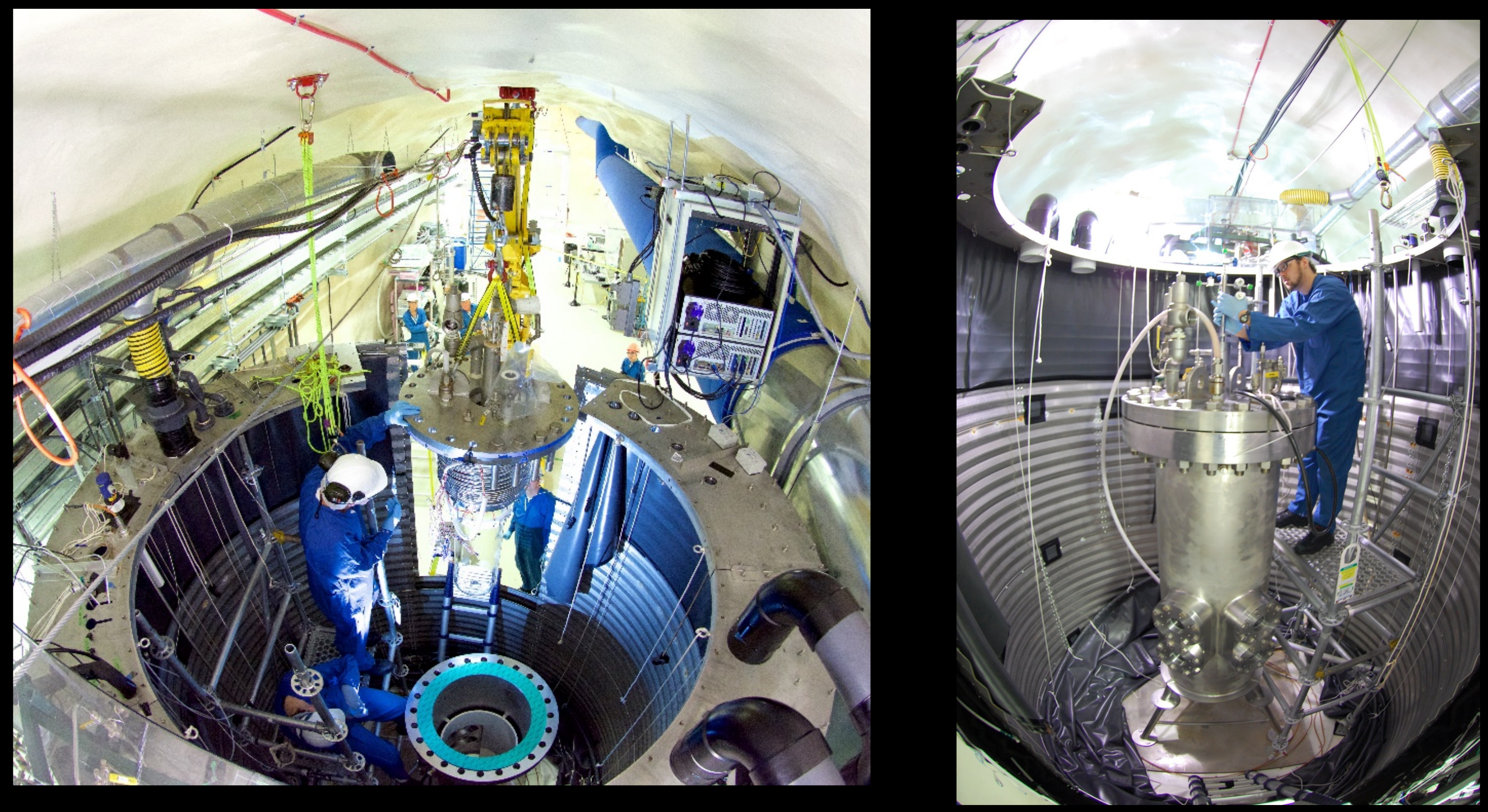

- Filled with 40 liters of C3F8 on June 30, 2016

- First physics run Nov 2016-Jan 2017 


\section{PICO-60 Blinding Strategy "Deafening"}

- Alpha events create single bulk bubbles that look like nuclear recoils except for acoustics

- Go deaf by not analyzing acoustic information for physics run

- Neutron calibrations to define nuclear recoil acceptance cuts

- All data to define fiducial volume/data cleaning/etc 


\section{Pre-unblinding}

- 106 bulk singles in WIMP search dataset

- Consistent with $\mathrm{Rn}$ decay rate in pre-WIMP search data

- $<20$ microBq $(<500 \mathrm{nBq} / \mathrm{kg})$

- Neutron backgrounds

- Not blinded to multiplicity, observe 3 multiples

- Multiple to single ratio is $3: 1$ (simulation and calibration)

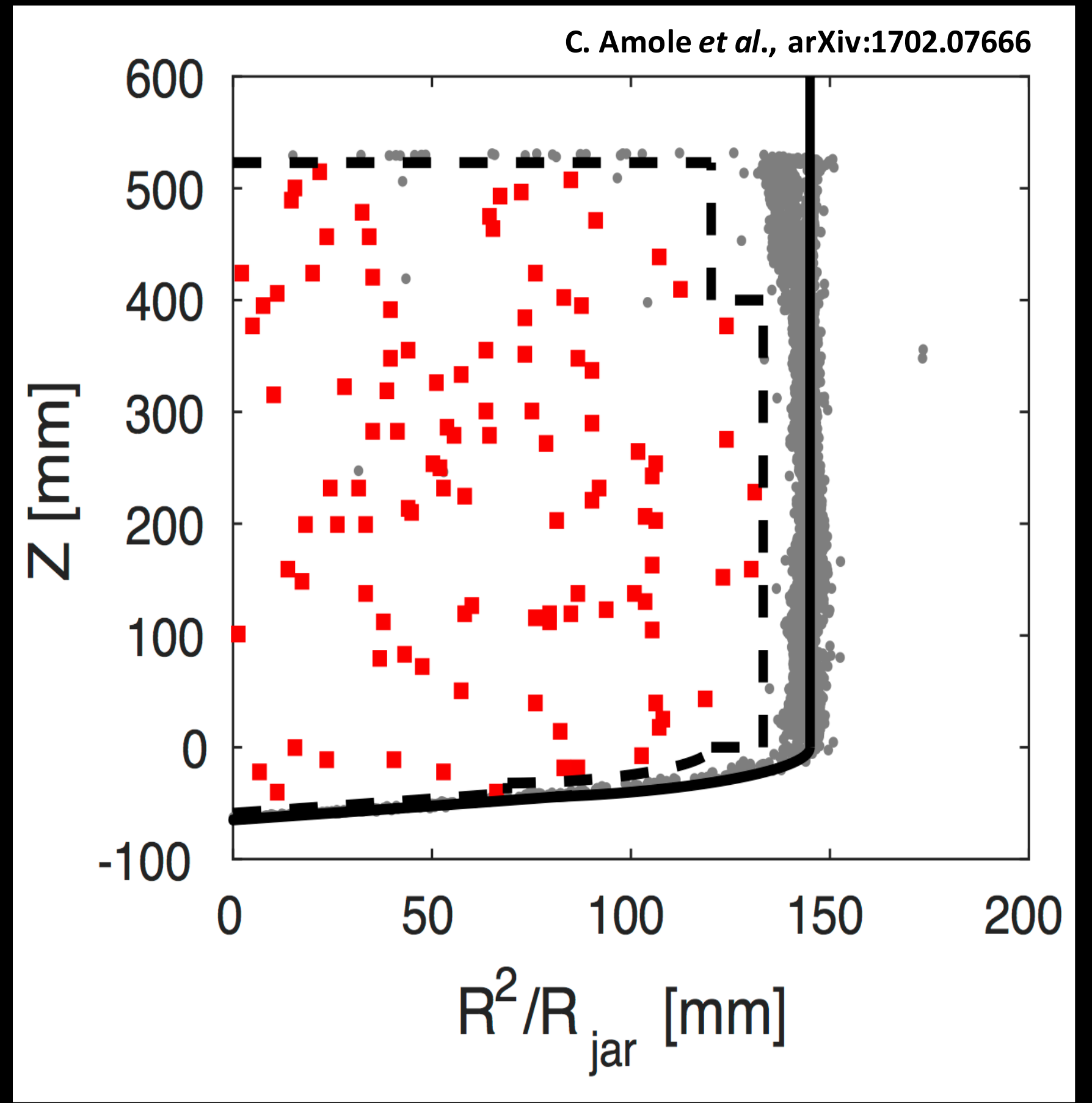




\section{Post-unblinding}

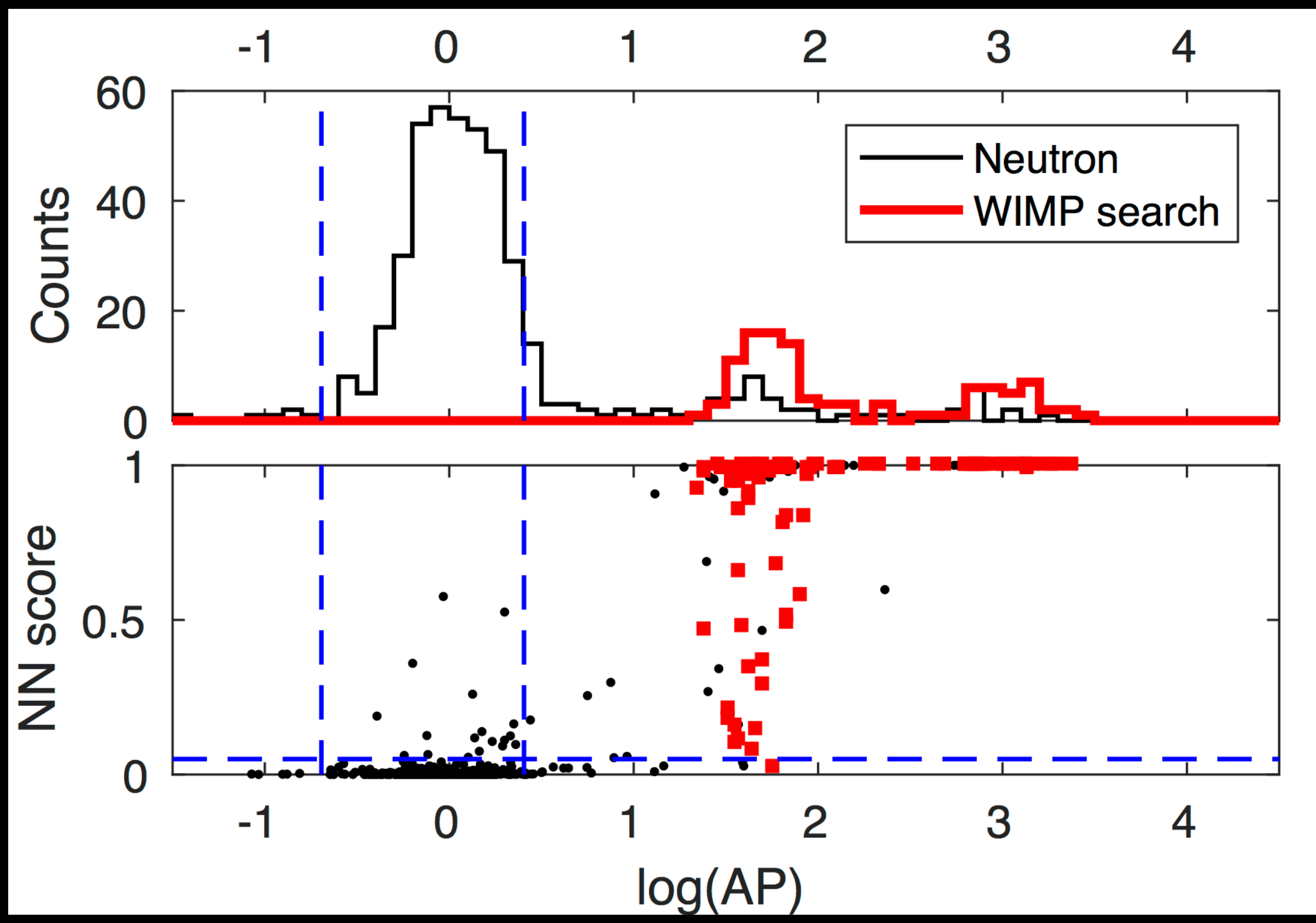

- No signal events! 


\section{PICO-60 Results}

\begin{tabular}{lcccc}
\hline \hline Dataset & Efficiency (\%) & Fiducial Mass (kg) & Exposure (kg-days) & No. of events \\
\hline Singles & $85.1 \pm 1.8$ & $45.7 \pm 0.5$ & $1167 \pm 28$ & 0 \\
Multiples & $99.4 \pm 0.1$ & $52.2 \pm 0.5$ & $1555 \pm 15$ & 3 \\
\hline \hline
\end{tabular}

TABLE I. Summary of the final number of events and exposure determination for singles and multiples in the 30.0 live-day WIMP search dataset of PICO-60 $\mathrm{C}_{3} \mathrm{~F}_{8}$ at $3.3 \mathrm{keV}$ thermodynamic threshold.

C. Amole et al., arXiv:1702.07666

- Multiples efficiency is higher because there are no acoustic cuts

- We simulate and measure a 3:I ratio of multiples to singles in neutron calibration data

- Of the 106 fiducial-bulk singles, none are consistent with nuclear recoil hypothesis

- No dark matter

- No anomalous background 


\section{PICO-60 Results}

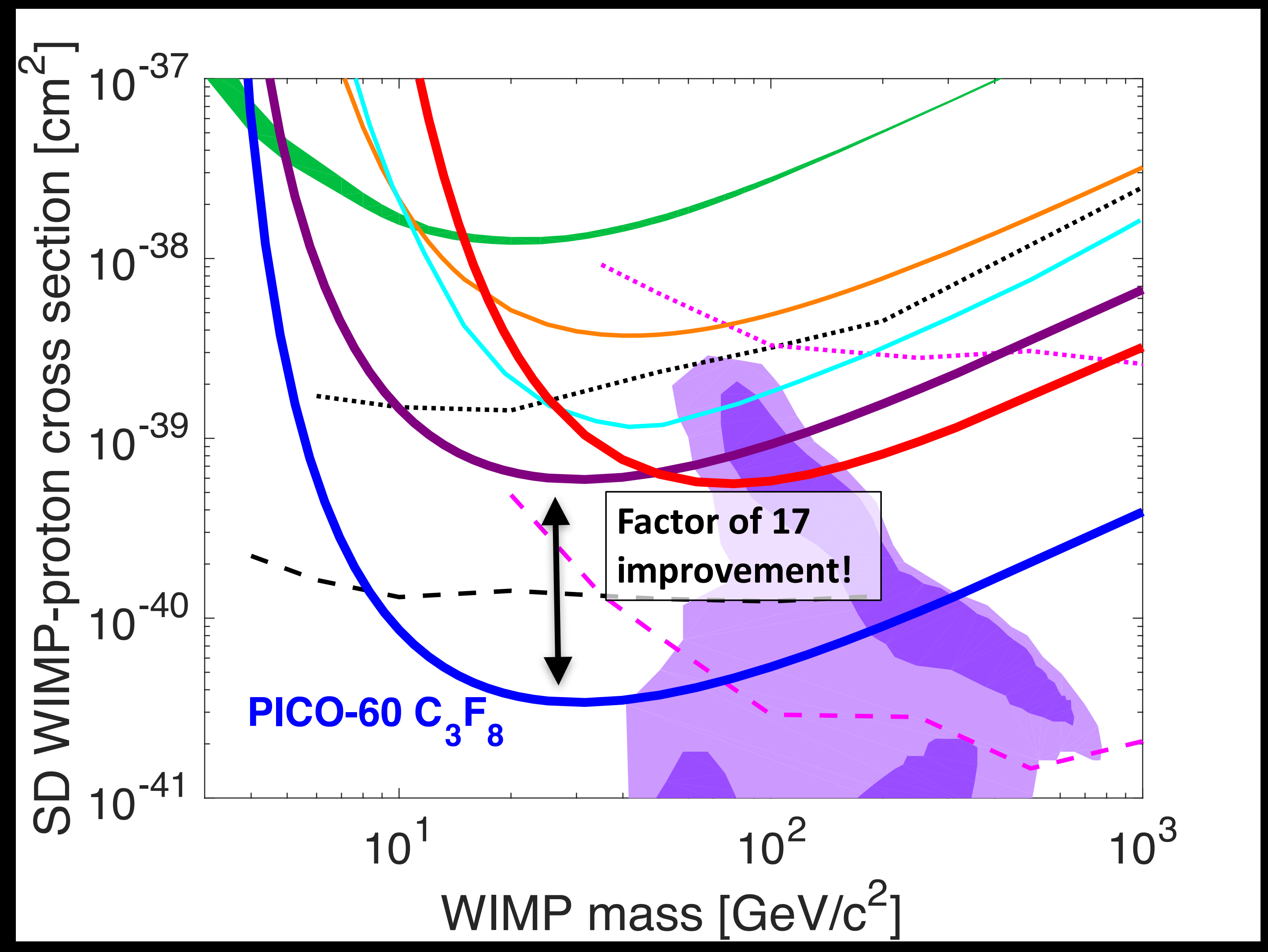




\section{Comparison to Collider}

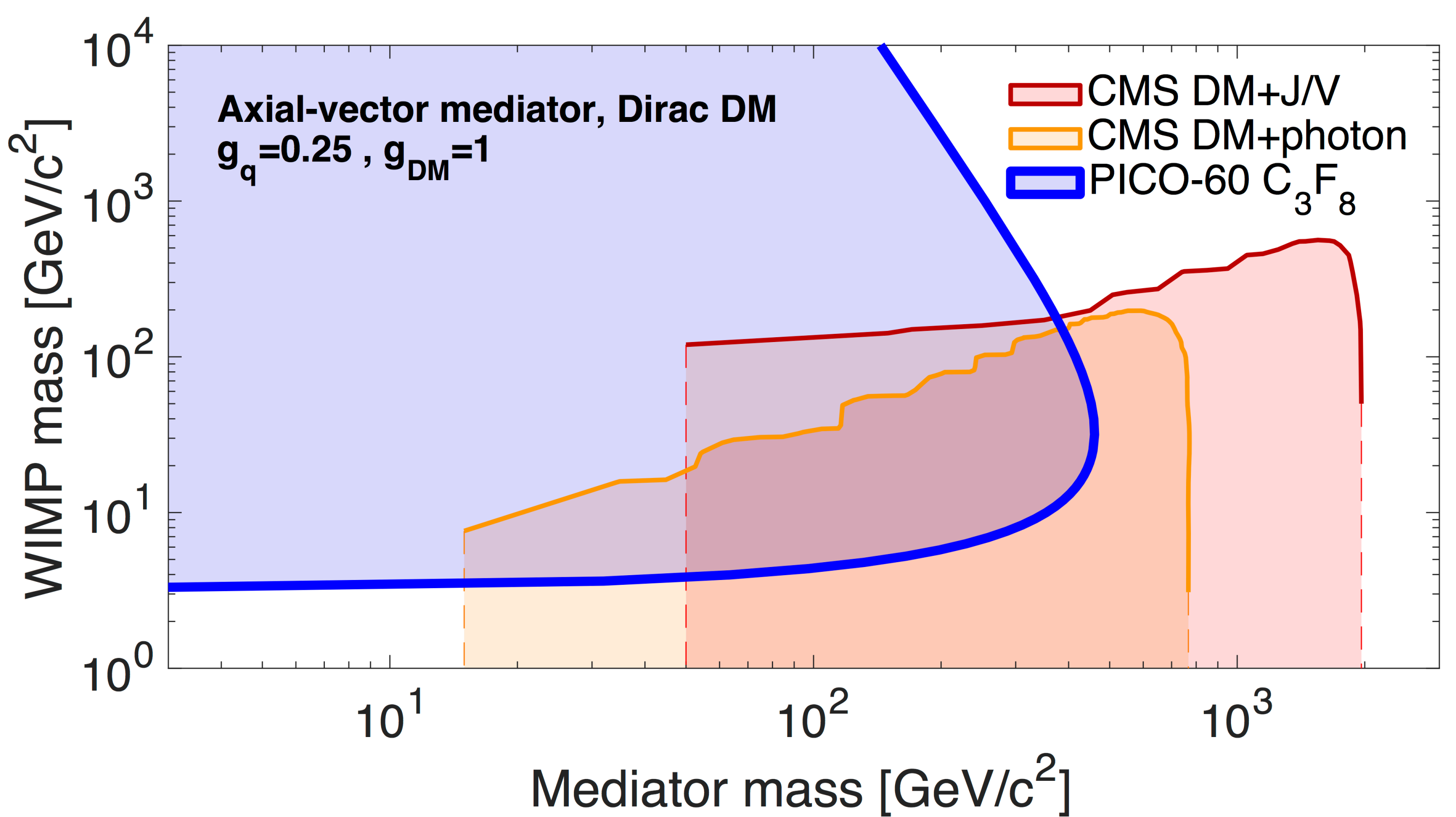

- Sensitivity to common model 


\section{PICO-60 Since January}

- Calibration runs at variety of thresholds

- Scanning gamma sensitivity

- 30 days of blind running at lower threshold

- Still "deaf" to acoustics

- Updating optical reconstruction algorithms to improve efficiency before opening the box 


\section{PICO-60 Decommissioning}

- Emptied and removed from water tank end of June

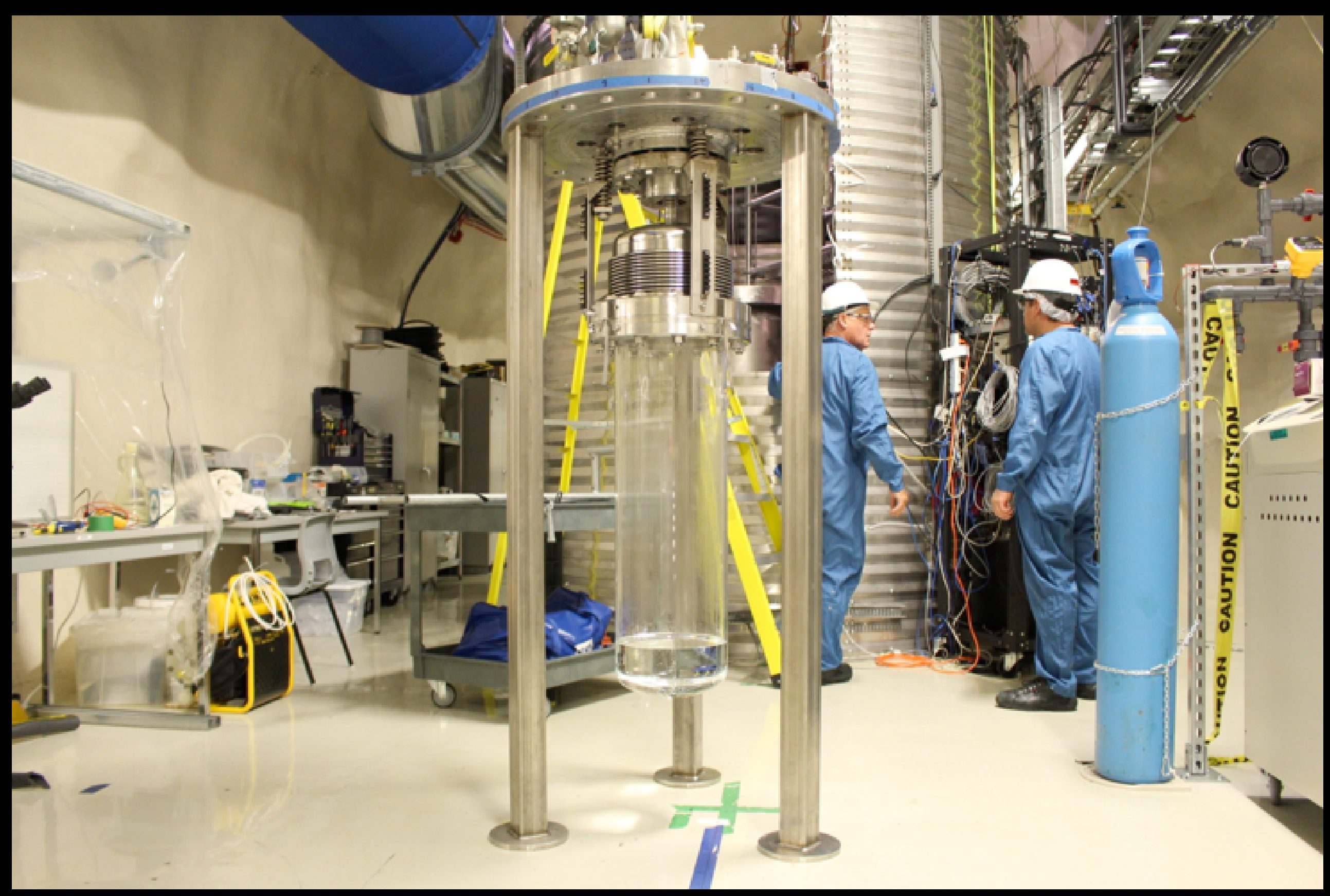

- Making room for PICO-40L 


\section{PICO-40L}

- We've shown that particulate contamination can create bulk bubbles

- Mechanism remains uncertain, but we believe it is the interaction of the particulate with the buffer fluid

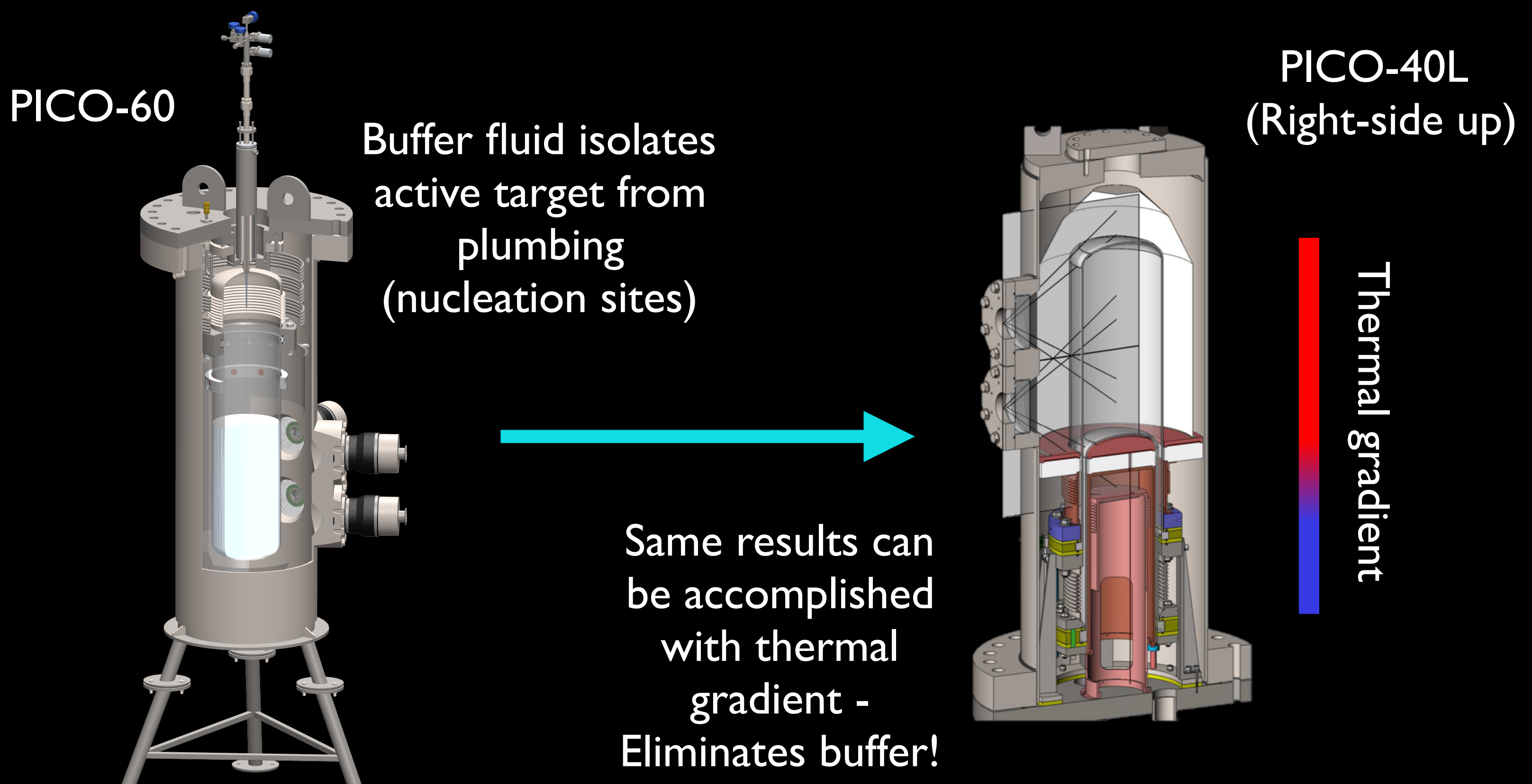




\section{PICO-40L}

- PICO-40L is first large scale RSU device

- Northwestern xenon bubble chamber

- Drexel C3F8 chamber

- Re-using PICO-60 water tank

- Increased physics reach

- Expected improvements in stability allow for lower threshold running

- Larger pressure vessel allows major neutron sources to be moved out reduction by factor $\sim 5$

- Commissioning this winter

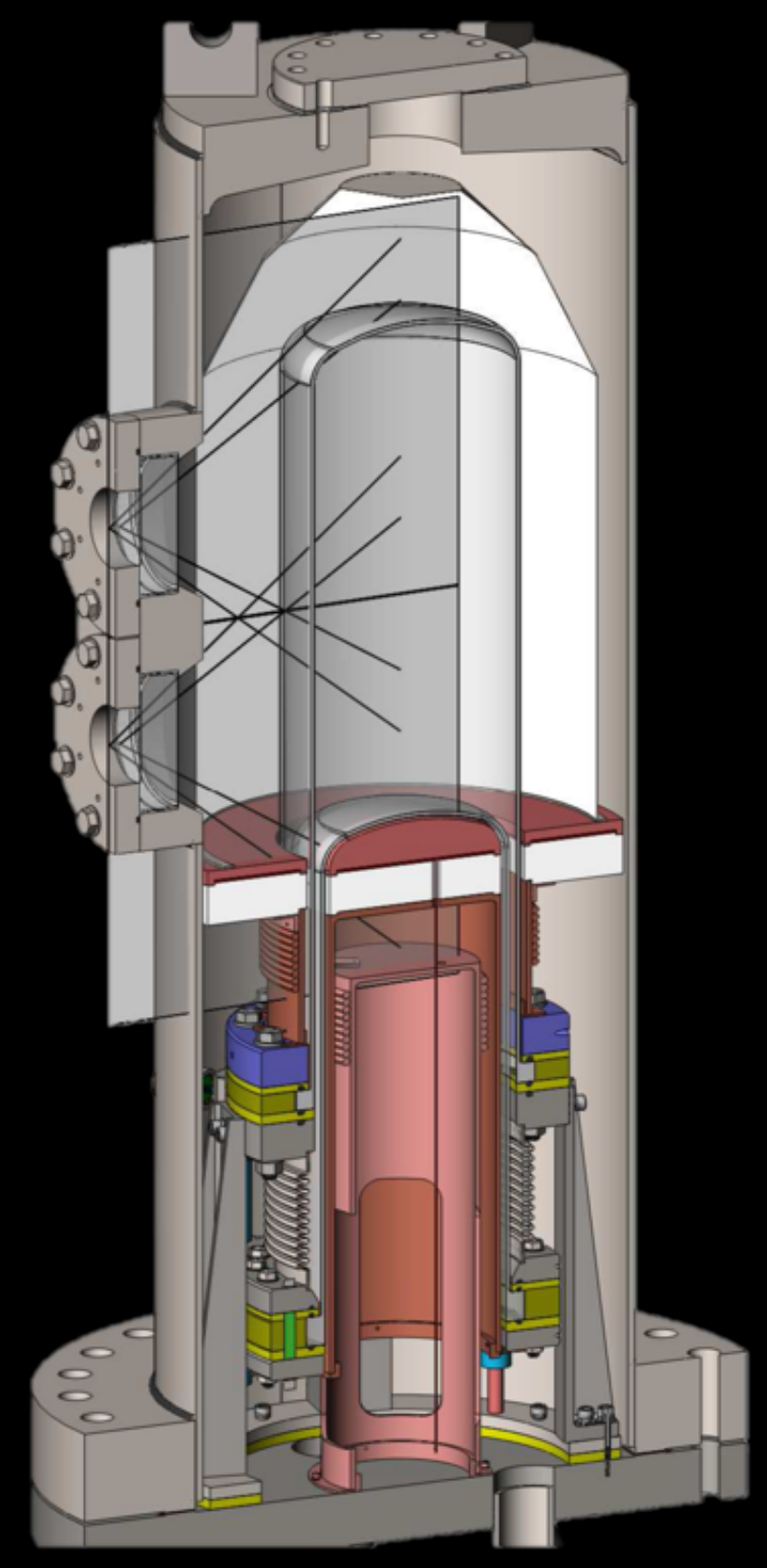




\section{PICO-40L}
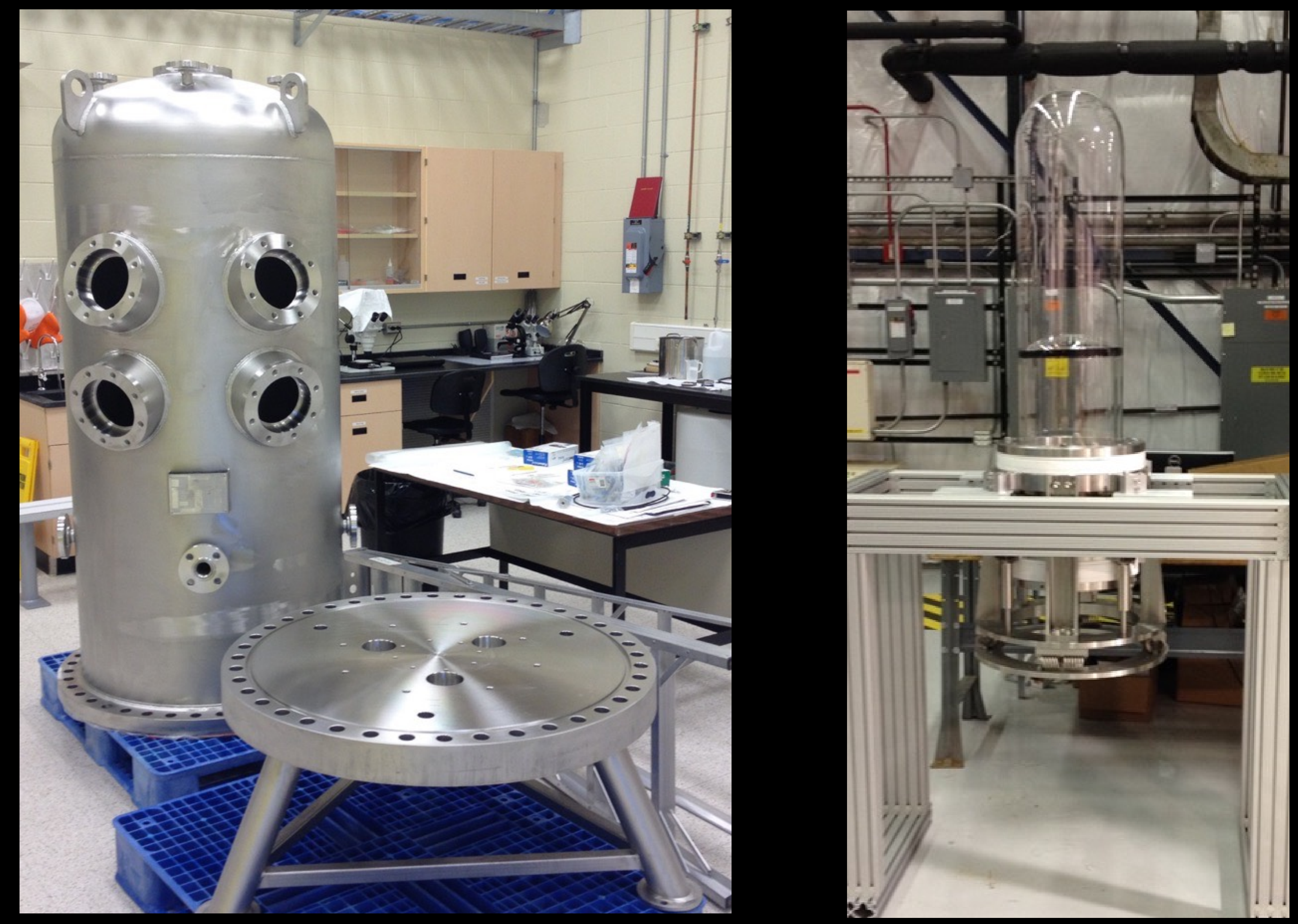


\section{PICO-40L}

- Pressure vessel arrived in May

- Cleaning of assembly on surface now ongoing

- Shipping underground this winter

- First data in January

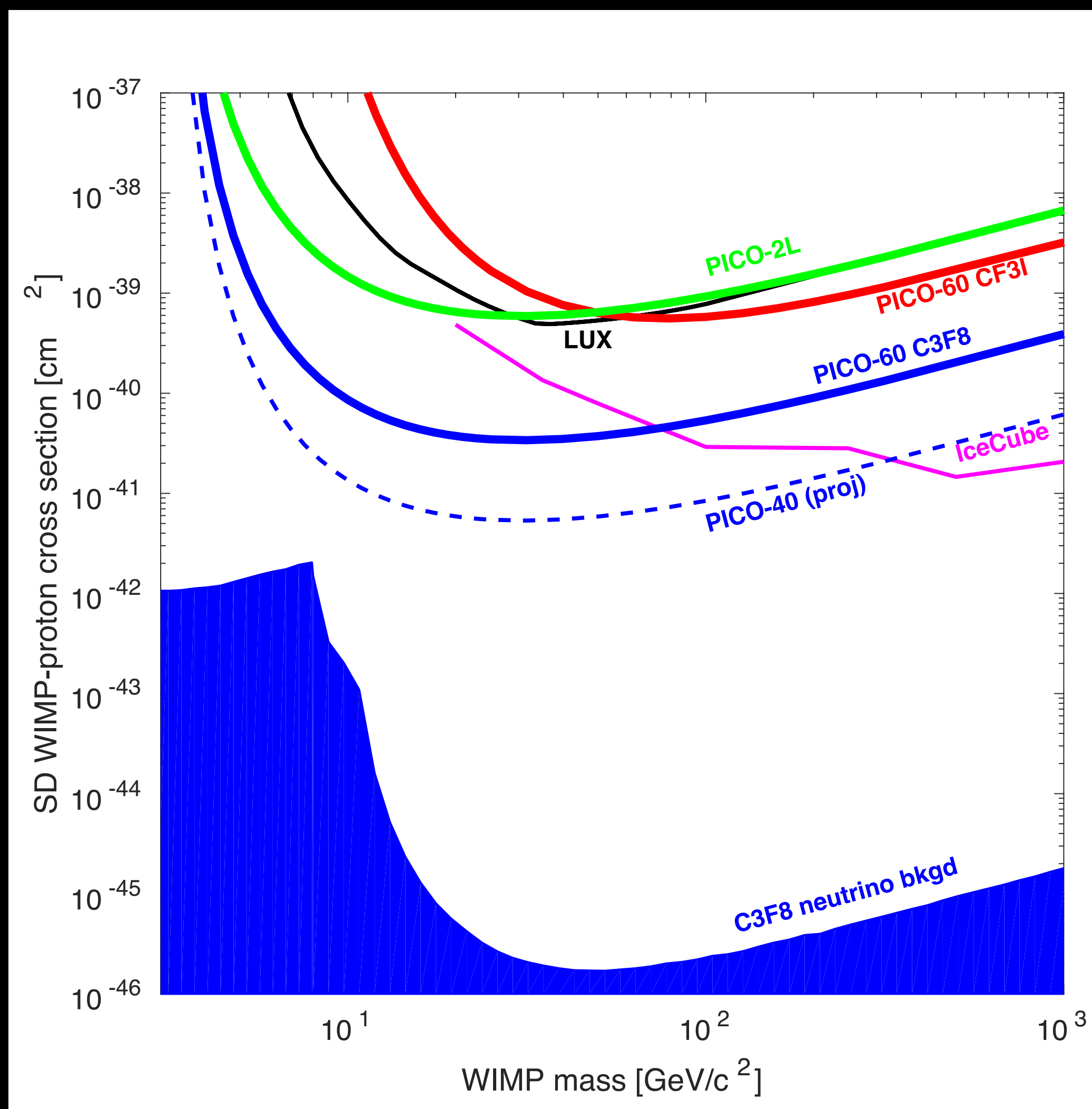




\section{Scaling to PICO-500}

- Background free at $\sim 50 \mathrm{~kg}$ scale

- PICO sensitivity (spin-dependent, proton) complementary to other areas of DM program

- At multi-tonne scale, fluorine better at SD coupling than than heavy nuclei

- Coherent neutrino scattering scales like $N^{\wedge} 2$, while SD sensitivity is "flat"

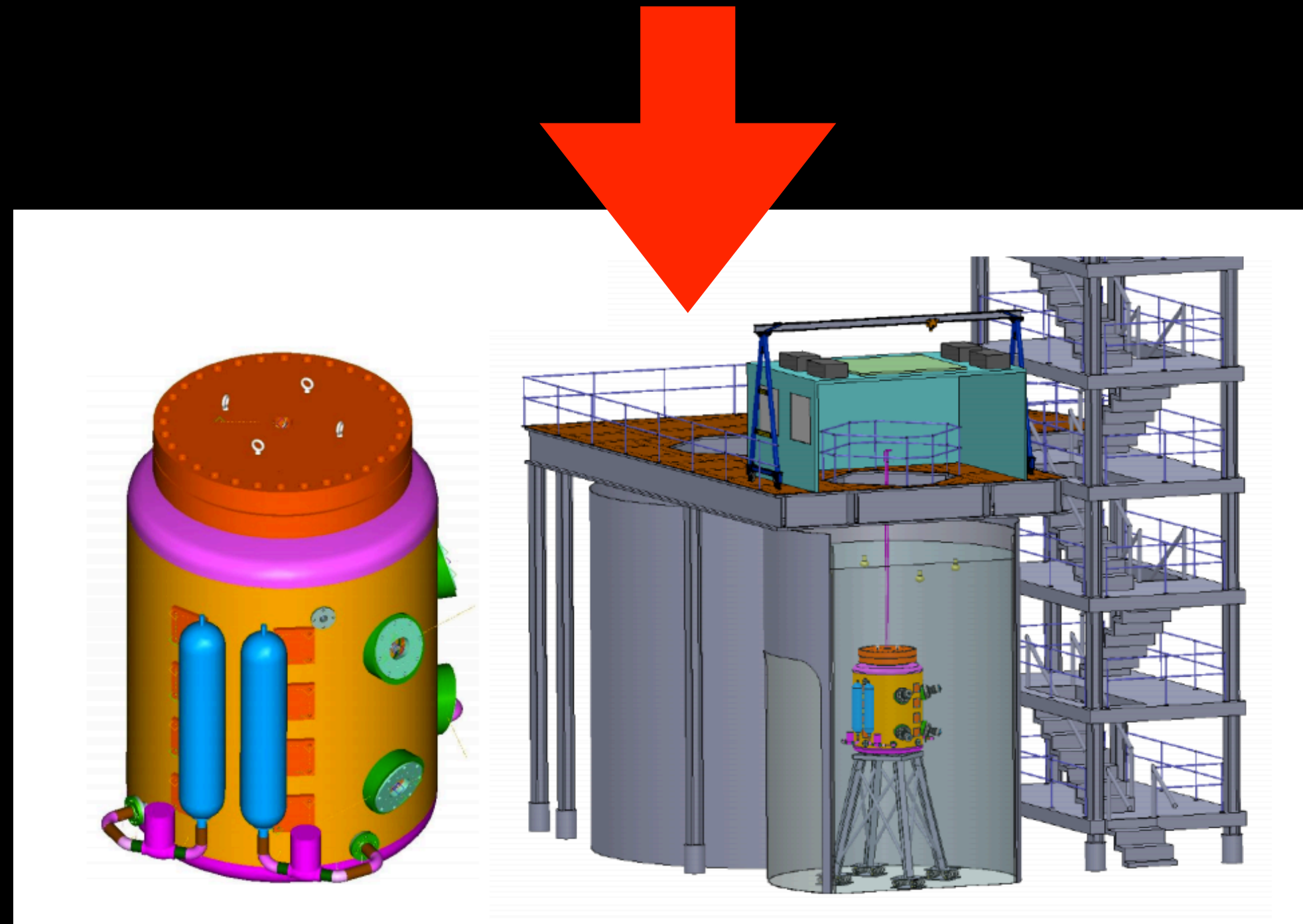




\section{Scaling to PICO-500}

- PICO-500 is proposal for ton-scale device to be built at SNOLAB

- Proposed project start in 2018

- Simulations and background control protocols are well underway

- Final detector design will depend on PICO-40L results

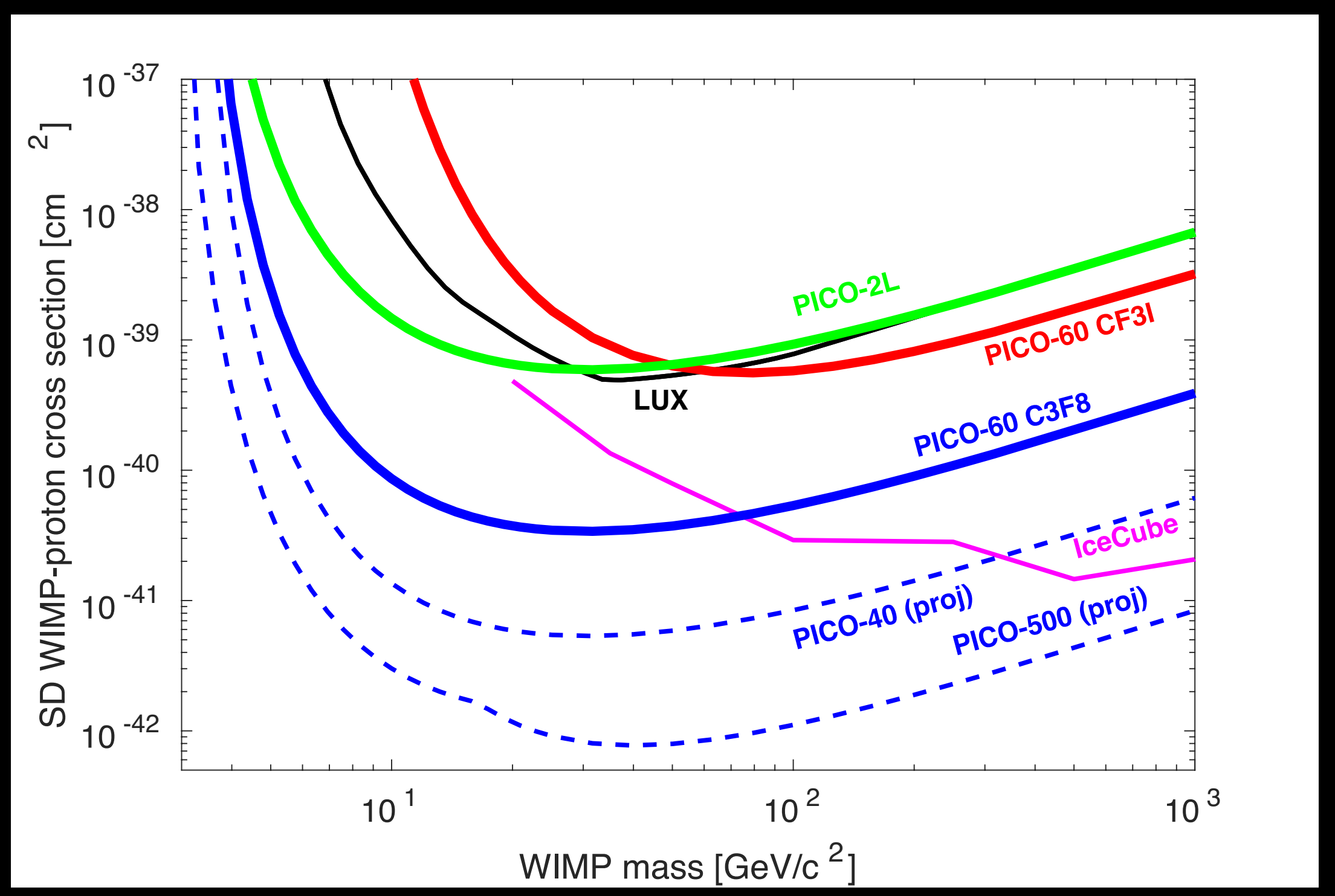




\section{Scaling to PICO-500}

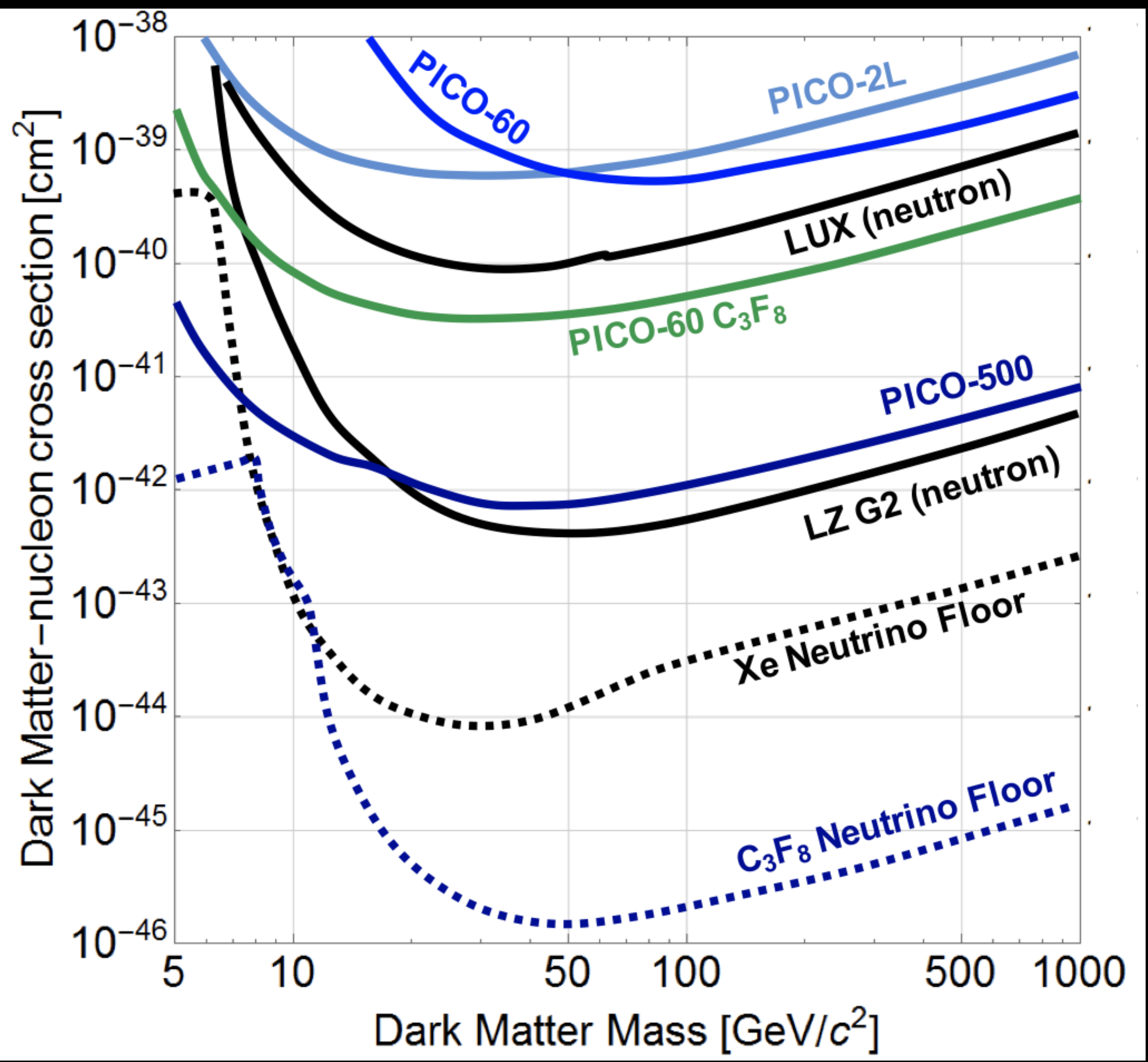




\section{Conclusion}

- PICO bubble chambers at the 40 liter/50 kg scale can be made background-free

- PICO setting world-leading constraints on spin-dependent WIMP proton coupling

- Moving on to the next phase of the program

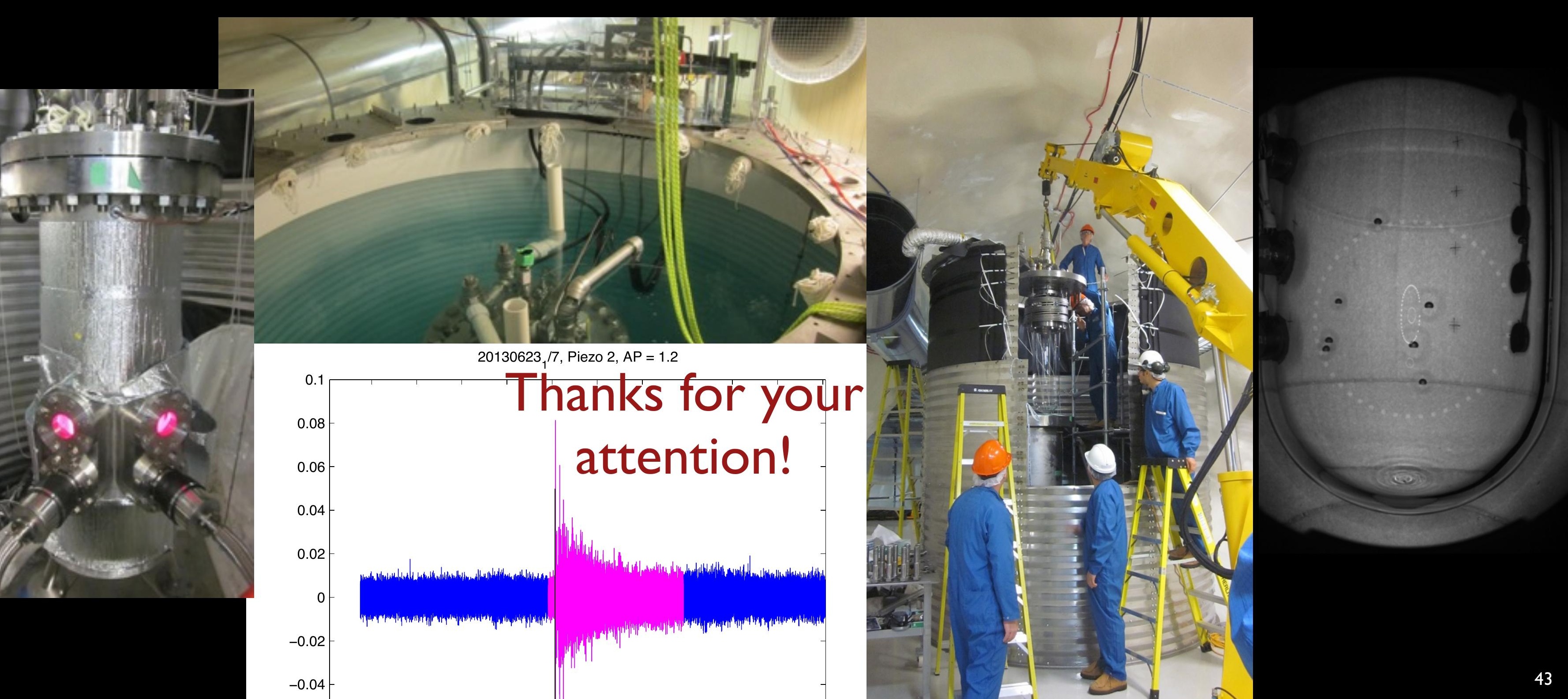




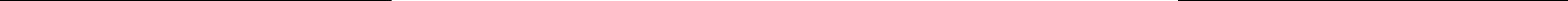




\section{Extra}




\section{SI vs. SD (vs. nuclear physics)}

- Spin-independent historically dominates the news because of the rate enhancement (xI6000 for an atom like xenon)

- True interaction is still unknown

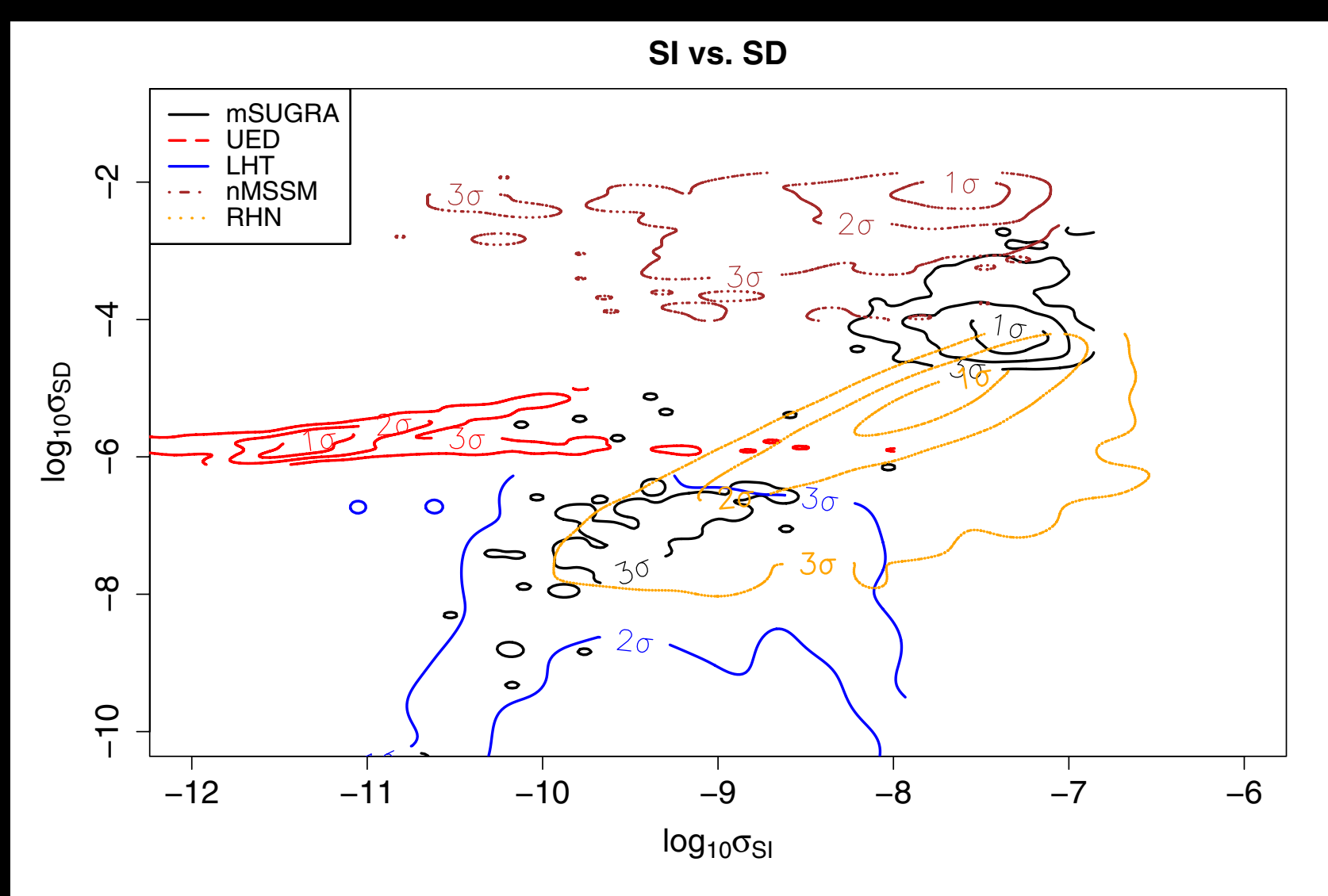

SD vs. SI cross section predictions for different models (Barger, PRD, 78 056007)

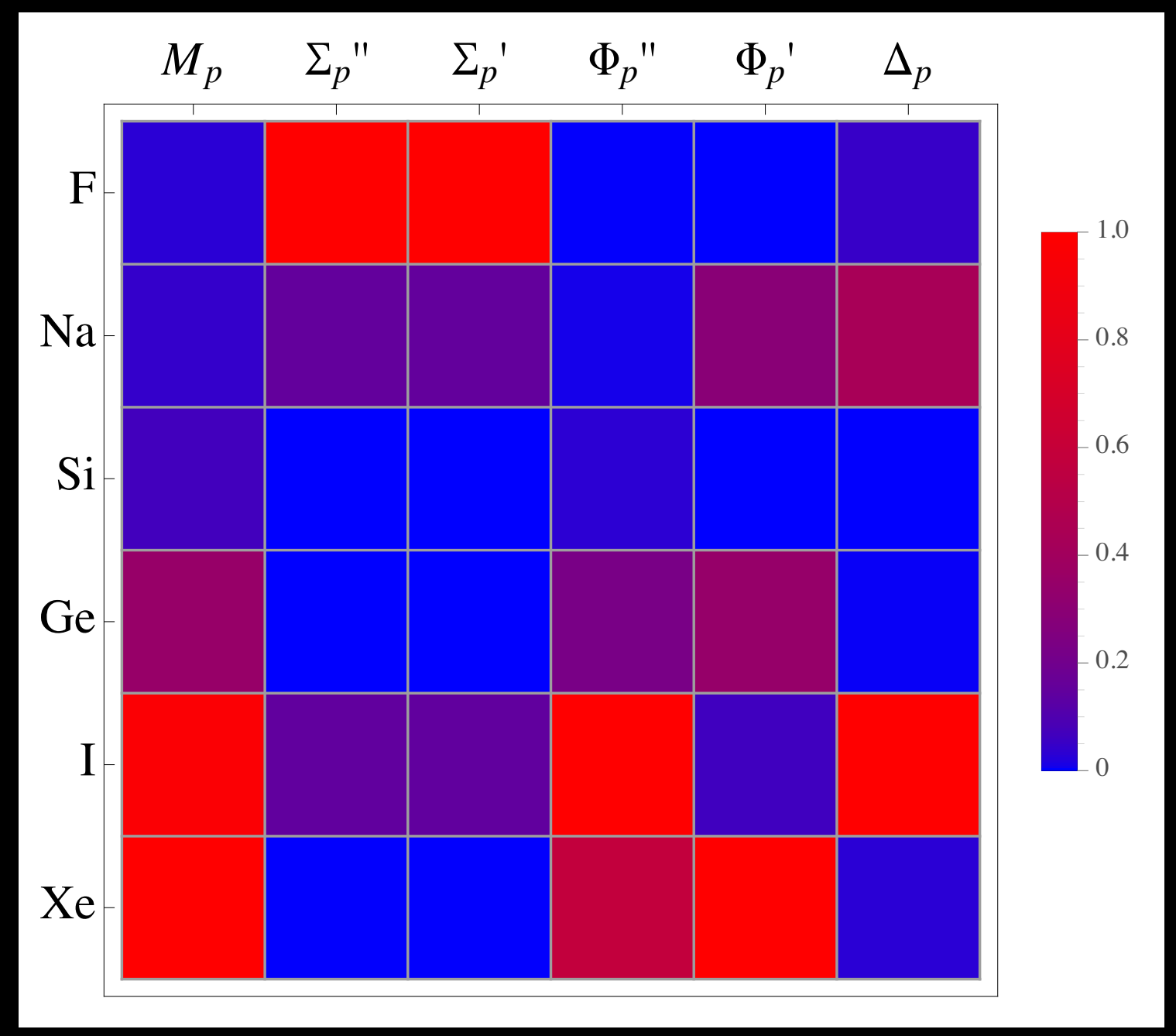

Sensitivity of different p-coupling operators to various nuclear targets

(from L. Fitzpatrick at INT Workshop, 20I4) 


\section{Why bubble chambers?}

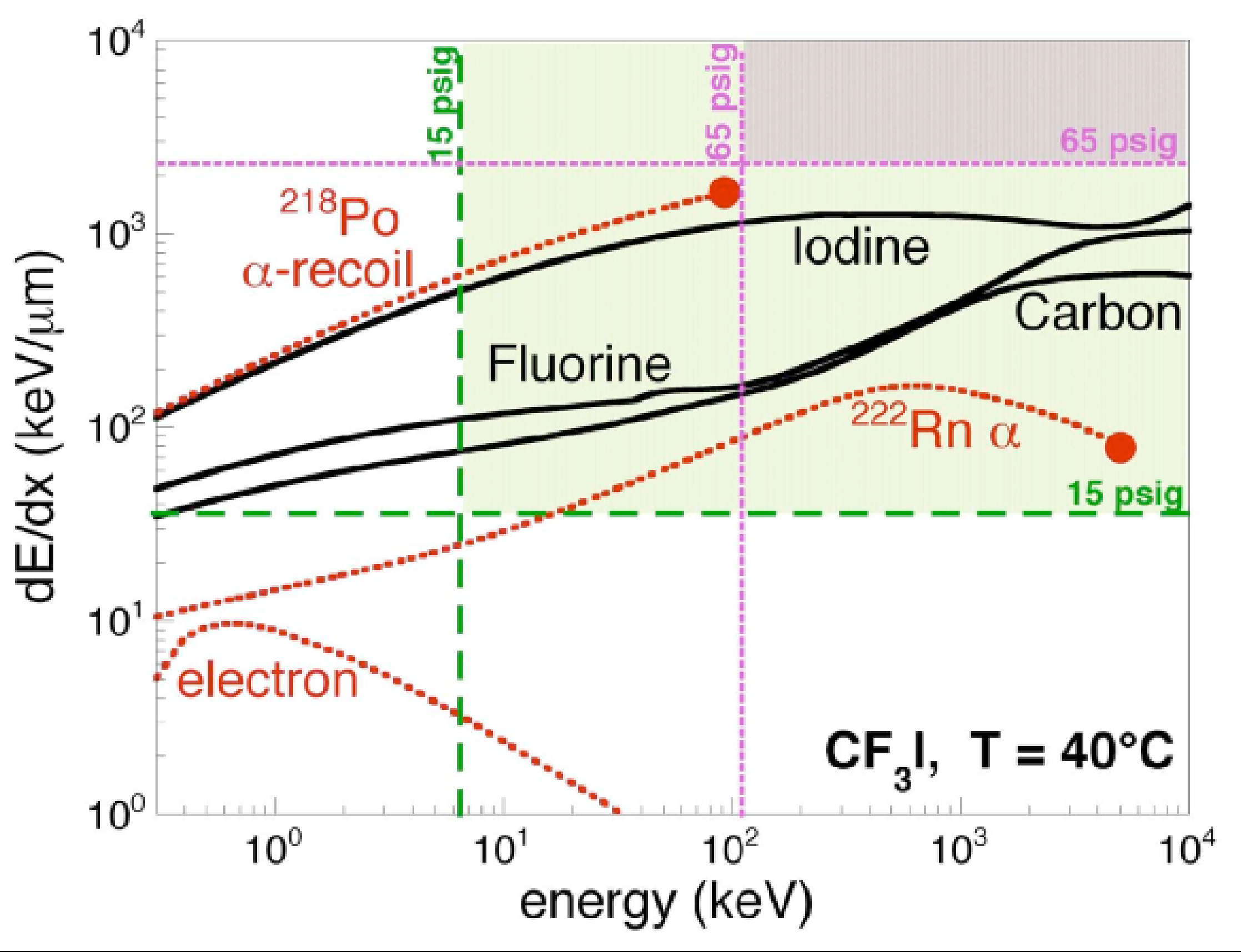




\section{Detour:Threshold and efficiency}

- Threshold determined from Seitz, Phys. of Fluids I, 2 (1958)

$$
\begin{gathered}
p_{v}-p_{l}=\frac{2 \sigma}{r_{c}} \\
E_{t h}=\underbrace{4 \pi r_{c}^{2}\left(\sigma-T \frac{\partial \sigma}{\partial T}\right)}_{\text {Surface energy }}+\left(\frac{4}{3} \pi r_{c}^{3} \rho_{v} h\right)
\end{gathered}
$$

- Energy deposition $E_{t h}$ within length $R_{c}$ will nucleate a bubble (Hot Spike model)

- Theory assumes a step function above threshold 